\title{
Chapitre 13 Le dehors du langage au dedans du dire : la « question » de la parole propre
}

\section{Introduction}

Il ne s'agit donc plus ici de « positionner » son discours parmi ceux des autres, dans un jeu narcissique de "petites différences »... ou de grands conflit, mais pour un sujet - qui ne l'est que d' « être parlant » - de se placer dans le langage, c'est-à-dire, dans la foncière altérité de celui-ci, d'y assurer le sentiment - nécessaire - de tenir une parole propre.

Ce qui se joue aux frontières intérieur/extérieur d'une parole ne peut être dissocié du caractère crucial, pour le sujet humain, aux plans interpénétrés du corps et du langage, du sentiment, au fondement de son identité, de constituer une entité distincte.

Pour approcher ce qui relève, non d'un donné de naissance, mais - pour le sujet en devenir, puis à maintenir - d'un mouvement d'instauration de « limites identifiantes », productrices d'un " effet de UN », on rappellera d'abord (1) la nécessité vitale et archaïque de la distinction dedans/dehors, dans la constitution d'une image de soi, au plan, pour le sujet en devenir, de son corps baigné de langage ; avant (2) de s'attacher à la contradiction propre au langage, d'un dedans " fait » de dehors, telle qu'elle apparaît pour l' « infans » entrant dans la parole, et dans les réflexions de théoriciens de l' « inappartenance foncière du langage », pour - derrière l'évidence d'un usage de l'instrument de communication disponible - reconnaître, à travers pathologies langagières ou expériences communes, ce qui se joue dans le fait d'accéder, ou non, ou mal... à une parole posée, dans l'altérité langagière d'où elle procède, comme " à soi », c'est-à-dire d'envisager la parole dans sa dimension de question.

\section{De l'indistinction primitive à l'image de l'un parmi d'autres}

\section{Descartes et Michaux}

Et comment est-ce que je pourrais nier que ces mains et ce corps-ci soient à moi ?

dit Descartes, ouvrant les Méditations par l'expression de cette évidence communément partagée... Mais c'est lui-même qui, aussitôt, vient troubler cette certitude 
raisonnable de la dissonance d'un «Si ce n'est peut-être... » qui, tout en la tenant à distance, fait place, dans la réalité humaine... à la déraison :

Si ce n'est peut-être que je me compare à ces insensés, de qui le cerveau est tellement troublé et offusqué par les noires vapeurs de la bile, qu'ils assurent constamment qu'ils sont des rois, lorsqu'ils sont très pauvres ; qu'ils sont vêtus d'or et de pourpre, lorsqu'ils sont tout nus ; ou s'imaginent être des cruches, ou avoir un corps de verre. Mais quoi ! ce sont des fous, et je ne serais pas moins extravagant, si je me réglais sur leurs exemples.

De fait, le sentiment d'un « corps à soi » n'est pas un donné « de naissance » : le caractère factuel de "séparation » que comporte la naissance ne suffit pas; il y faut la constitution, par chacun, d'une image de son corps, distincte et cohésive où, dans un processus d'individuation - susceptible de rencontrer des difficultés, de connaître des échecs... - puisse se fonder son « moi ». Laissons la parole à Henri Michaux pour évoquer - non pas en opposition à Descartes mais en amont du plan, où celui-ci se place, des certitudes normalement acquises -, d'après la radicale expérience personnelle où s’ancre son œuvre, le parcours à accomplir parmi «Les grandes épreuves de l'esprit ${ }^{1}$, pour atteindre à la nécessaire délimitation de soi :

L'enfant dans son tout premier âge confondait la main, la tête, le sein, la mère et lui-même dans une sphérique sphérifiante impression globale qui n'avait pas de fin. Seul le sommeil revenait souvent, revenait l'entourer, mais le sommeil est-ce une frontière ?

Etrange planète, que chacun de nous a été. L'homme est un enfant qui a mis une vie à se restreindre, à se limiter, à s'éprouver, à se voir limité, à s'accepter limité. Adulte, il y est parvenu, presque parvenu. (p. 147)

\subsection{L’idée - corporelle - de nous-mêmes, avec « un dedans et un dehors ».}

Les mots de Michaux entrent en résonance avec ceux - indistinction, fusion, symbiose... vs limite, frontière, séparation, démarcation, partage, enveloppe... - qui, à travers tout le champ psychanalytique, portent, avec constance, une pensée de la limite comme inséparable du devenir humain : non celle d'une sagesse appelant à se garder de l'excès, de l'hybris..., mais celle, primitive, existentielle, de l'instauration progressive, dans l'indistinction des origines, d'un partage dedans/ dehors, intérieur/extérieur, solidaire de l'émergence du moi ${ }^{2}$ que - en écho à la

1 Gallimard, Paris, 1966, repris in 1994..

2 E. Porge (2012 : 84) évoquant « la distinction entre le dedans et le dehors, laquelle ne s'opère qu'une fois que le moi a pris forme », par opposition à « une altérité régnant sans partage, dans l'extase et l'angoisse ». 
conception freudienne des instances psychiques de 1923 d'un Moi « avant tout corporel $»^{3}$ - l'on retrouve dans l'évocation par Gribinski (2013: 19) de la réalité psychique comme supposant que

notre idée de nous-même ait quelque chose de corporel, avec un dedans et un dehors.

L’aventure, au fondement archaïque du moi, de cette séparation individuante, est, certes, affaire de corps, mais pas, on l'a dit, celle d'un corps qui serait « laissé à lui-même » si l'on peut dire : séparé du corps de la mère il l'est à la minute où se fait la coupure du cordon ombilical... La sortie hors de la « sauvagerie maternelle $»^{4}$, de cet espace psychique primitif « d'avant l'ordre de la langue, avant les commencements de l'apprentissage du monde $»^{5}$ se fera par l'émergence - éprouvée " par ses bords » - d'une image du corps propre, nécessaire pour que le chaos de l'indistinction mère-enfant "se civilise dans la différence qui fait d'un enfant un "autre" »".

\section{Un corps " exposé au langage »}

On doit noter que les caractérisations, fréquentes, du moment fusionnel mère-enfant comme « temps d'avant le langage » sont trompeusement approximatives car il ne peut s'agir, pour l'infans, plongé dans le langage, que du temps d'avant son entrée parlante - passant par l'ordre de la langue - dans celui-ci. Selon une thèse majeure de Lacan, il n'y a pas pour l'humain - quelque soit le moment où l'on se situe de sa maturation - $\mathrm{d}^{\prime}$ " en deçà du langage " $^{7}$ :

[...] l'homme qui naît à l'existence a d'abord affaire au langage ; c'est une donnée. Il y est même pris avant sa naissance, n'a-t-il pas un état civil ? Oui, l'enfant à naître est déjà, de

3 In « Le Moi et le Ça » : « [le moi] est en quelque sorte une continuation de la différenciation superficielle », « le moi est avant tout un moi corporel », cité in Roudinesco et al. (1997 : 681), qui commentent : «Aussi faut-il l'appréhender comme une projection mentale de la surface du corps. "

4 Titre d'un ouvrage d'A. Dufourmantelle (2016).

5 Dufourmantelle (2016:18) : » espace [dit l'auteur], que l'on peut seulement approcher par défaut dans ses contours, ses plaintes, ses effets dans la mythologie qui en recueille la voix, dans certaines destinées, dans le repli des œuvres [...], moment où l'enfant est à peine séparé de la mère, que Mélanie Klein, Bion, Winnicott ou Bettelheim ont magistralement décrit [et] reste pourtant une énigme qui échappe au discernement des soignants ».

6 Ibid. p. 19.

7 Cf. l'analyse par C. Soler (2008 : 30 sq.) de l'incidence du langage sur la perception, notant que « cette thèse heurte "la foi perceptive" que tous les êtres parlant partagent » (p. 36). 
bout en bout, cerné dans ce hamac de langage qui le reçoit et en même temps l'emprisonne (Lacan 1957, entretien).

Nombreuses sont les expressions - en référence explicite à Lacan ou non - de cette conception de l'humain comme non séparable du langage ; par exemple celle d'A. Dufourmantelle (2001: 255), dans la métaphore de «l'enfant [...] habité par le langage dès sa conception », ou, radicalement chez Jean Oury $(2006: 12,13)$ lorsqu'il évoque, dans le sillage de Lacan

le corps tissé de signifiants, l'existence tissée de paroles, marques dans le corps des signifiants [...],

Et, pour s'en tenir à l'expérience de tout un chacun - que l'on retrouvera ci-dessous quand il s'agira pour le sujet devenu parlant d'énoncer une parole propre on sait bien que, avant qu'il ne parle lui-même, l'enfant a été un infans, et un corps à qui, de qui, autour de qui..., à la place de qui, on a parlé, par là baigné, rythmé, nourri, bercé, tissé, meurtri, ... de langage.

Ainsi, le corps dont l'image comme distinct apparaît en corrélation avec l'émergence individuante du moi est un corps " parlé », ou, si l'on peut dire, un corps « langagié ». Et l'instauration des frontières dedans/dehors qui le délimite est indissociable du mode sur lequel le sujet est « exposé » au langage ; c'est ce qui s'impose à suivre le parcours que F. Dolto ${ }^{8}$ retrace, dans ses étapes et ses écueils, de la "figuration du corps » : distincte du « schéma corporel » qui est une « réalité de fait », l’image du corps s'élabore, prend forme dans le milieu de la parole environnante.

\subsection{Pensées de l'instauration d'une limite identifiante}

Dans l'immense ensemble des travaux consacrés à ce chemin de différenciation première, je retiendrai trois conceptualisations : «l'objet transitionnel » de Winnicott, le « stade du miroir » de Lacan, le « moi-peau » de D. Anzieu. Il est clair que, dans un champ traversé par des différences théoriques qui ne sont pas mineures, la notion de limite ne relève pas d'une conceptualisation unique, et que l'usage « banalisé »" que j'en fais ici - à juxtaposer des traitements qu'il n'est pas question

8 Dolto (1984), L'image inconsciente du corps.

9 Je pense à un passage - dans le texte théoriquement aigu de C. Soler (2008) - où, évoquant, de façon critique, diverses interprétations de phénomènes psychotiques en termes de « défaut dans le repérage des frontières », l'auteur conclut sur une forme « d'accord » possible dès lors qu'il se 
d'articuler théoriquement ${ }^{10}$ - se situe à un niveau « infrathéorique » : j'espère cependant pouvoir raisonnablement y dégager quelques uns des traits par lesquels peut se penser, au plan subjectif, la vitale instauration d'une limite - frontière, contour, enveloppe... -, susceptible d'éclairer, ultérieurement, au plan du discours, les enjeux subjectifs du tracé de contour de la RDA.

\subsubsection{Avant la séparation : un espace de transition}

C'est négativement que la notion d'objet transitionnel ${ }^{11}$ dessine celle de limite : amorce d'une distinction à venir, à un moment où l'enfant est «à peine séparé » de sa mère, cet objet auquel, entre quatre et douze mois, l'enfant s'attache - bout de chiffon, coin de couverture... " véritable fétiche tactile et odorant, associé au téter d'un ou deux doigts ${ }^{12}$ - relève, en effet

[d']un champ intermédiaire d'expérience dont l'enfant n'a à justifier l'appartenance ni à la réalité intérieure, ni à la réalité extérieure (et partagée) ${ }^{13}$

qui constitue pour lui un espace de passage,

de transition de la relation fusionnelle (non-moi) vers une symbolisation de la relation objectale (moi) $)^{14}$

\subsubsection{L'« infans » au miroir : « moi » aux côtés d'« un autre »}

En revanche, c'est foncièrement de la survenue d'une limite de soi, comme distinct d'un autre, qu'il s'agit dans le moment (de première ébauche du $\mathrm{Moi}^{15}$ ) que J. Lacan a appelé «stade du miroir » ", étape cruciale de la « figuration du corps » à travers laquelle l'enfant, face au miroir et aux côtés d'un autre, témoin, découvre sa propre image :

situe, en deçà, à un moindre niveau d'exigence théorique : « [...] il est clair en tout cas qu'il y a effectivement un type de phénomènes qui évoquent une question de frontières » (p. 74).

$10 \mathrm{Cf}$. note 31 et note 35 ci-dessous, les rencontres sur fond de discordance théorique touchant à la conception du moi et du sujet.

11 Cf. D.W. Winnicott $(1953,1971)$, voir les articles « objet transitionnel » des dictionnaires de psychanalyse : Laplanche et al. (1968) et Roudinesco et al. (1997).

12 Dolto (1984 : 254) : situé « entre le pouce et l'ours en peluche » dit Winnicott.

13 Winnicott, cité in Laplanche et al. (1968: 296).

14 Roudinesco et al. (1997: 741).

15 Le Moi, comme instance - imaginaire - du sujet, $c f$. chap. 10.3, p. 410.

16 Lacan (1949), voir les articles «stade du miroir» des dictionnaires de psychanalyse de Laplanche et al. (1968) et de Roudinesco et al. (1997). 
En se reconnaissant à travers cette image, l'enfant récupère ainsi la dispersion du corps morcelé en une totalité unifiée qui est la représentation du corps propre. L'image du corps est donc structurante pour l'identité du sujet qui y réalise son identification primordiale (Dor 1985 : 101).

De cette expérience - fondatrice du narcissisme primaire - à partir de laquelle, dit F. Dolto (1984:154) «ce ne sera plus jamais comme avant», je retiendrai quelques traits :

- la fonction d'assurance - de protection - contre les fantasmes de corps morcelé, envahi... que l'enfant trouve dans l'image corporelle d'unité cohésive à laquelle, sur un mode « jubilatoire » dit Lacan ${ }^{17}$, il s'identifie ;

- cette image de soi n'est fondatrice qu'en tant qu'elle passe par l'autre, à la fois $v u$ dans le miroir (semblable et différent) et regardé, témoin, parlant, de ce jeu d'images : il ne suffit pas que l'enfant se voie, image aperçue dans, dit Dolto (1984: 148)

un miroir plan [qui] à rien ne sert [s'il n'est expérience] d'un miroir de son être dans l'autre (idt).

L'expérience spéculaire est donc, certes, d'ordre visuel, mais indissociablement relationnelle et symbolique ${ }^{18}$, comme en témoigne, selon $\operatorname{Lacan}^{19}$,

[...] le geste par quoi l'enfant au miroir, se retournant vers celui qui le porte, en appelle du regard au témoin qui décante, de la vérifier, la reconnaissance de l'image [...].

- l'image de la totalité unifiée dont, en passant par l'autre, l'enfant s'assure dans le miroir, est une image identifiante, porteuse de distinction :

L’enfant ne peut donc plus, dans la réalité, à partir de l'expérience scopique partagée avec autrui, se confondre $[. . .]^{20}$.

17 Cf. par exemple, in Lacan (1947, repris in 1966 : 185) : « l'assomption triomphante de l'image avec la mimique jubilatoire qui l'accompagne et la complaisance ludique dans le contrôle de l'identification spéculaire », $c f$. encore Schneider (2013: 63) : « la toute puissance de l'enfant découvrant son image dans le miroir ».

18 Point sur lequel insiste F. Dolto (1984) dans sa présentation (p. 147-163) de ce qui se joue « au miroir », soulignant que l'enfant découvre que «son visage est visible pour autrui comme l'est pour lui le visage des autres » (p. 157) et le convainc [...] qu'il est semblable aux autres humains, un parmi eux » (p. 158). Cf. Prieur (1986 : 298) : « Le moment de formation du moi, de reconnaissance et d'identification à son moi est [...] corrélatif du surgissement de l'étranger. »

19 Lacan (1966: 678) cité par E. Porge (2012: 74).

20 Dolto (1985 : 154), qui précise : « ni se confondre avec les autres, ni se prendre pour (“croire vraiment être") un avion... ou un cheval..., condition pour accéder plus tard aux jeux fictionnels du conditionnel : je serais etc. ». 


\subsubsection{L'interface du Moi-peau}

Une autre pensée de la limite comme élément crucial sur le chemin de l'individuation - menant, selon les termes de F. Dolto ${ }^{21}$, du « circuit court » d'un corps à corps de l'enfant à la mère au « circuit long de communication de psychisme à psychisme » - est celle du Moi-peau, développée par D. Anzieu²2.

La constitution par l'infans d'un Moi-peau va de pair avec l'instauration d'un moi distinct : répondant, pour l'infans, au besoin d'une « enveloppe narcissique », le moi-peau relève

[d'] une figuration [...] au cours des phases précoces de son développement pour se représenter lui-même comme Moi contenant les contenus psychiques, à partir de son expérience de la surface du corps. ${ }^{23}$

Les trois fonctions principales qui sont reconnues à cette construction psychique - « enté[e] sur le corps propre ${ }^{24}$ - s'ancrent dans « l'expérience de la surface cutanée $e^{25} »$ :

- celle d'une contenance, protectrice d'un dedans individuel, par son caractère d'enveloppe ;

- celle, associée à la première dans le processus d'individuation, d'une délimitation, distinction, séparation, barrière... d'avec l'extériorité ;

- celle, enfin - qui fait la stimulante spécificité du Moi-peau comme conception de la limite - d'interface ${ }^{26}$.

Ni mur, ni ligne infranchissable, la frontière du moi-peau est dotée à la fois de porosité et d'épaisseur, pensée - à partir de la double réceptivité de la peau aux sensations internes et externes - comme espace à double face, lieu de rencontre, d'échange, de communication entre un intérieur et un extérieur (qui, pour « communiquer » doivent préalablement avoir été distinguées).

21 Dolto (1984: 83, 102)

22 Anzieu (1974, 1985), la notion, développée en 1985 dans l'ouvrage intitulé « Le Moi-Peau », apparaît en 1974, comme élément d'une publication collective significativement intitulée « Le dehors et le dedans ".

23 Anzieu (1985 : 61), cf. aussi (ibid. : 59) «L'infans acquiert la perception de la peau comme surface à l'occasion des expériences de contact de son corps avec le corps de la mère [... parvenant ainsi] à la notion d'une limite entre l'extérieur et l'intérieur. »

24 Gribinski (2013:34).

25 Freud (1923), évoqué par Anzieu (1985/95 : 120).

26 Anzieu (1985/95 : 84 sq.) : « Particularités du Moi-peau considéré comme interface ». 
D. Anzieu ${ }^{27}$ marque nettement, pour cette dimension bi-face du moi-peau, le caractère précurseur de la notion tôt élaborée par Freud ${ }^{28}$ de « barrière de contact », associée à des fonctions de « tamis » ou de « filtre » pour les stimulations externes et internes. L'intérêt de cette conception psychique de la «peau », permettant de penser le fait humain crucial de la distinction, non pas comme une disjonction - résultat d'une coupure « chirurgicale »... - mais comme résultant, dans un espace vivant, de la rencontre de forces hétérogènes (endo- et exogènes), a été souvent relevé, dès lors que (se réclamant d'une fidélité à Freud ${ }^{29}$ ) l'ancrage dans le corps des réalités psychiques était reconnu comme essentiel : ainsi Gribinski ${ }^{30}$, déjà convoqué, ou A. Dufourmantelle qui, rappelant la notion de « membrane de délimitation » proposée par Winnicott, reformule le moi-peau en « lien de peau entre le dedans et le dehors ». Citons aussi, entrant en résonance avec ce qui précède - mais sans référence à la notion de Moi-peau ${ }^{31}$ - ces mots de O. Grignon dans «États de corps » :

[...] la peau n'est pas un sac, ce n'est pas une barrière entre l'intérieur et l'extérieur. Au contraire c'est un lieu troué, un lieu d'échange, un espace d'attraction et de répulsion. La peau vivante, c'est d'abord la caresse. (Grignon $2017: 240$ )

Notons encore, dans une perspective de " sémiotique du corps », la réflexion que J. Fontanille consacre au « modèle du Moi-peau », insistant sur la spécificité « topologique » de cette

membrane entre deux domaines [...] lieu critique où des forces et des mouvements aboutissent, passent, sont bloqués, bifurquent, etc. (Fontanille 2002 : 202, idt)

soulignant le caractère de contrôle de cette instance interface ${ }^{32}$.

27 ibid. p. 98-104.

28 Dans sa correspondance avec Fliess (1895) publiée (1956) sous le titre « Esquisse d'une psychologie scientifique ».

29 Cf. Anzieu (1985/95 : 119) évoquant comme « spécifiquement freudien » le principe selon lequel « toute fonction psychique se développe par appui sur une fonction corporelle dont elle transpose le fonctionnement sur le plan mental ».

30 (2013 : 28-38), au chapitre « Anatomie animique » qui, s'ouvrant sur un commentaire du catalogue d'une exposition intitulée «L'âme du corps ", débouche sur la notion élaborée par D. Anzieu, dont il reprend la litanie des «mots de peau »: "Tout être vivant a une peau, une écorce, tunique, enveloppe, carapace, membrane, méninge, armure, pellicule, cloison, plèvre... Quant à la liste des synonymes de membrane, elle est considérable : [...]. » Anzieu (1985/95 : 35).

31 Hétérogène théoriquement au cadre lacanien affirmé de 0 . Grignon.

32 Contrôle auquel répond, je pense, au plan du Discours, le caractère de surplomb métadiscursif de la RDA sur lequel on revient au chap. 14. 
Remarque 1 : Peau redessinée ou redoublée : le tatouage et la « robe ». Il faut noter l'extension des fonctions de l'image de la peau comme enveloppe, barrière de contact... à ce qui se surajoute à sa surface nue :

- ce qui s'y imprime, comme les incisions, scarifications, tatouages, peintures, maquillage, etc., pratiques de dessin ou d'écriture du corps, dont la fonction symbolique a été largement explorée par les anthropologues et les observateurs des sociétés urbaines contemporaines, et que Anzieu ${ }^{33}$ évoque rapidement comme un « développement de la fonction du Moi-peau »;

- ce qui la redouble, vêtement, piercing, bijoux... dont, dans un cadre lacanien, E. Lemoine-Luccioni ${ }^{34}$ explore la fonction de « seconde peau ${ }^{35}$ qui « tend à refaire l'enveloppe : à refaire un dedans et un dehors ${ }^{36}$, réassurant l'effet de un (limite, cohésif) de l'image " au miroir », c'est-à-dire de cette forme dont le sujet

a besoin de façon vitale, parce que l'Autre est là, en qui le corps se perdrait, n'était ce contour imaginaire que le sujet se donne $[. . .]^{37}$.

\subsubsection{Fonctions, enjeux et troubles « de la limite »}

Les hypothèses, conceptualisations, évoquées ci-dessus, touchant la nécessité pour le sujet humain de l'instauration d'une frontière dedans/dehors, sont indissociables - en tant qu'elles sont issues de leur observation et qu'elles visent à permettre une réponse thérapeutique - des troubles psychiques renvoyables à un défaut de cette frontière ${ }^{38}$.

Si Freud a caractérisé « la psychose comme un trouble entre le moi et le monde extérieur ${ }^{39}$, c'est une constante de spécifier ce trouble en " manque de limites » et d'observer que ce que l'on nomme aujourd'hui les « états limites » (ou borderline), c'est-à-dire à la limite entre les phénomènes névrotiques et la « folie » psychotique, relèvent de « pathologies dans lesquelles précisément les limites ne sont pas définies ${ }^{40}$.

33 (1985/95: 128).

34 E. Lemoine-Luccioni (1983) La Robe - essai psychanalytique sur le vêtement.

35 Expression qu'elle introduit et commente ainsi : « Je reparlerai volontiers ici de seconde peau et même de "moi-peau" si cette notion ne traînait derrière elle des présupposés théoriques où je ne me reconnais pas. » (p. 131)

36 ibid., p. 81.

37 ibid., p. 41, je rappelle que l'Autre, avec une majuscule, irreprésentable, s'oppose à l'autre, celui par lequel, au contraire, l'un peut se poser comme différent, $c f$. « le lieu de l'Autre » in Lacan 1966, p. 898-9), ou Roudinesco et al. (1997: 83) : «Autre voir ci-dessous p. 509 ».

38 Ainsi le parcours de F. Dolto (1984) des étapes de la « figuration du corps » se déroule-t-il continûment sur le double fil de l'observation clinique et de l'élaboration théorique.

39 Roudinesco et al. (1997: 848).

40 Schneider (1985 : 293) ; cf. aussi Anzieu (1995:29) : «En fait ces malades souffrent d'un manque de limites »; cf. Roudinesco et al. $(1997: 260):$ " Etats limites ». 
«Questions de frontière ", « problème des frontières du corps », "précarité des frontières entre dedans et dehors », « abolition de l'écart constitutif entre le dedans et le dehors ", " séparation impossible », " indifférenciation », " symbiose $» . .$. reviennent comme un leit-motiv dans les approches (par ailleurs non-consensuelles) des formes variées de psychose. Et à ces «troubles de la frontière » correspondent - à tous les degrés de gravité, de la fragilité passagère à la catastrophe - des altérations des diverses fonctions qu'assure la frontière :

- distinction, individuation, identification dont le trouble laisse le sujet, victime de " défaut identitaire et d'indétermination de son être $~^{41}$, aux prises avec les menaces d'intrusion, de dépersonnalisation, de « voix $»^{42}$;

- sentiment d'unité et de cohésion, dont la fragilisation livre le sujet à la persécution de "ses doubles » et aux angoisses de " corps morcelé $»^{43}$;

- caractère interface de la séparation dont le ratage condamne le sujet au repli derrière une barrière isolante, étanche - telle la « forteresse ${ }^{44}$ de l'enfant autiste, interdisant, parfois jusqu'au mutisme, tout contact.

Ainsi, à titre d'exemple, parmi tant d'autres, ces deux évocations de troubles douloureux de la limite : celle de Robert Schumann auquel parmi ses suggestives attributions fictives d'œuvres, P. Bayard ${ }^{45}$ attribue la paternité du Cri de Münch, en manière d'approcher la folie du musicien, l'angoisse hallucinée qui le conduira au suicide, en proie à une « absence de séparation entre l'intérieur et l'extérieur », une " dissolution terrifiante des limites », dans une souffrance de " perte fondamentale d'identité » où s'engloutit le sujet. Ou, dans une autre tonalité, celle de R.M. Rilke et de sa « fragilité narcissique » où se retrouve - exprimé en tourments physiques et nerveux accompagnant la création - cet enjeu de la limite, du rapport au monde extérieur qu'évoque F. Gantheret ${ }^{46}$ en termes d'enveloppe, ou de peau, fragilisée :

41 Soler (2008: 130-131) ; cf. Chabert (2011 : 10) : « la précarité des frontières entre dedans et dehors, une porosité des enveloppes [...] expose aux intrusions [...] ».

42 Cf. ci-dessous 2.3.2, p. 522.

43 Cf. par exemple G. Pankow, clinicienne de la psychose, évoquant chez les psychotiques « des zones de destruction de l'image du corps », leur interdisant " de reconnaître un lien dynamique entre la partie et la totalité du corps ", perte du sentiment d'unité qu'illustre le cas, tragique, de la patiente éprouvant sa tête comme détachée de son corps (2006 : 113 sq. : « la statue décapitée [...]»).

44 Pour reprendre la métaphore de B. Bettelheim (1969) : « La forteresse vide. L’autisme infantile et la naissance du soi ».

45 Bayard (2010).

46 Gantheret F. (1996), Moi, monde, mots, chap. 1. 
La peau est usée, peu fiable : [...] l'unité de l'enveloppe n'est pas assurée,

et d'identité en danger, de ce que « la peau ne peut [la] sauvegarder ${ }^{47}$.

Loin de la linguistique ou de l'analyse de discours, ce parcours, certes un peu long, apporte aux notions (si souvent convoquées ci-dessus ${ }^{48}$ comme s'imposant descriptivement pour rendre compte des faits observés de RDA dans les discours) de frontière, de tracé dissimilateur, bases d'« identité verbale », de " figuration différentielle »..., la mise en perspective de ce qu'il en est, au plan - premier - de l'avènement d'un Moi (comme instance du sujet) « par ses bords ».

Parmi les traits à même d'ancrer « par les fondations » ce qui se joue, subjectivement, au plan du Dire, dans les frontières placées par la RDA, on peut retenir :

- le caractère mortifère de l'indistinction,

- et, corrélativement, celui, vital, du tracé de frontières identifiantes,

- la nécessaire mise en jeu différenciatrice d'un autre,

- la structure d'interface intérieur/extérieur de la frontière ;

ceci en portant l'accent sur deux caractéristiques de cette individuation :

- qu'elle n'est pas un donné, de naissance, mais le fait d'un processus, impliquant le langage, d'établissement de « bords »,

- que la séparation, imaginaire, qui en résulte n'est pas de l'ordre du « mur », mais, on l'a dit, un lieu, vivant, de contact et d'échange.

Et on soulignera que l'instauration de cette frontière

- n'est pas de l'ordre du tout ou rien, réussi/raté, mais qu'elle connaît des degrés, des zones de moindre solidité... ${ }^{49}$

- n'est pas non plus intangible, établie une fois pour toutes, à un certain stade du développement : elle évolue, se modifie, peut, dans son histoire, rencontrer des fluctuations passagères, des accidents, et que, par conséquent, cette frontière est, pour le sujet, continûment à maintenir, à faire vivre, à réassurer tout au long de la vie ${ }^{50}$.

47 ibid., p. 34, 35.

48 Chap. 11 et 12.

49 Cf., note 43 les « zones de destruction » évoquées par G. Pankow. »

50 Que l'on pense à Michaux (1, p. 493.) « presque parvenu », adulte, à « s'éprouver limité ». Ainsi, Anzieu, après avoir évoqué des thérapies d'« enveloppes de secours » mises en œuvre 
La construction psychique de ce « un parmi d'autres ${ }^{51}$ qu'est un sujet repose sur l'équilibre - précaire, vivant, toujours à refaire - d'un « être avec ${ }^{52}$ - à l'écart de la fusion autant que de la séparation radicale : de cet « être avec » on envisagera (chap. 14), au plan du langage et du discours, la RDA comme une des modalités.

C'est sur cette base qu'on se posera, au plan du langage ${ }^{53}$, la question $d u$ sentiment pour l'énonciateur « d'avoir une parole propre », c'est-à-dire du « comment » en discours, dans la parole, se joue « le mouvement très archaïque de recherche des limites $»^{54}$, comment parvient à s'établir la nécessaire frontière du dedans/dehors, assurant une image du " propre », alors que - contrairement au corps qui se « sépare ", de fait, à la naissance - le « dedans » de la parole est d'emblée, et irrévocablement, constitué de dehors.

\section{La contradiction fondatrice du langage : le « dehors » au " dedans " du dire}

Nous n'atteignons jamais l'homme séparé du langage [...]. C’est dans et par le langage que l'homme se constitue comme sujet. (idt) ${ }^{55}$

Ces propositions ancrent la réflexion de Benveniste sur « la subjectivité dans le langage »; pour lui, la « définition » de l'homme passe par le langage : et son " homme parlant $»^{56}$ n'est pas un homme " doué » de parole, mais - à l'instar $\mathrm{du}$ " parlêtre » de Lacan - homme de ce qu'il est parlant. Et, " être de langage », c'est dans notre parole que réside - y compris à notre insu -notre vérité la plus singulière.

pour restaurer des frontières gravement défaillantes, observe que « C'est là le grossissement d'un phénomène constatable chez les gens normaux qui ont besoin de reconfirmer périodiquement par des expériences concrètes leur sentiment de base d'un Moi-peau » (1985/1995 : 137).

51 Pour reprendre le titre de l'ouvrage de D. Vasse (1978).

52 Cf. J. Oury (2006 : 13) évoquant comme dimension où se joue notre existence, celle de " "l'avec" énigmatique".

53 Rappelons qu'il ne s'agit pas là de passer - en termes d'images que s'en fait le sujet - du plan du corps à celui du langage : il n'y a jamais eu de corps hors du langage, c'est d'un corps « exposé » au langage, on l'a dit, que le sujet se fait une image ; c'est de passer du corps (langagié) muet au corps parlant qu'il s'agit.

54 Schneider (1985 : 119-120) rappelant cette donnée à propos de faits de discours.

55 Benveniste (1966: 259)

56 ibid. «C'est un homme parlant que nous trouvons dans le monde [...], et le langage enseigne la définition même de l'homme». 
Pourtant, notre parole n'est pas «à nous » sur le mode où l'est notre corps : il n'est pas besoin de rejoindre les « insensés » évoqués par Descartes pour s'interroger sur l'appartenance des mots que nous disons.

De fait, la parole est le lieu, paradoxal, d'une essentielle contradiction : porteuse de la singularité du sujet dans le moment de son énonciation " propre », elle relève en même temps de cette « extériorité interne » reconnue comme une loi du langage - dialogique ou interdiscursive, $c f$. chap. 10. - qui, constitutivement, " déporte » la parole vers « de l'autre ».

Là où le Moi-corporel se fait en se séparant, se détachant psychiquement de la symbiose originelle, la parole qui advient - qui « prend corps » pourrait-on dire - ne se sépare pas de l'environnement langagier dans lequel, et dont, elle est faite.

De cette contradiction ${ }^{57}$ on évoquera, au plan subjectif, quelques facettes :

(1) comment elle est, comme " greffe », au cœur de l'advenue au langage de l'infans ;

(2) comment, depuis des points de vue théoriques divers, se formule le constat ou la plainte dont elle est l'objet pour le sujet parlant ;

(3) comment elle peut être « vécue », diversement dans l'expérience individuelle qu'en font les sujets.

\subsection{La « greffe » de l'entrée dans le langage : don et capture}

\subsubsection{Du vivant « dé-naturé » par le langage ${ }^{58}$}

Dans «La barque silencieuse »P. Quignard revient sur ce qui « fait source » à son écriture - la faille qu'inscrit en nous, nous arrachant, vitalement, à la « terrible extase infante », l'accès à la parole :

Le mot français d'enfance est extraordinaire. Il vient du latin in-fantia. Il veut dire en français a-parlance. Il renvoie à un état initial, non social, qui fait source en chacun de nous et dans lequel nous n'avons pas acquis notre langue. Nous sommes du non-parlant qui doit apprendre la langue sur les lèvres des proches. Aussi, quoi que nous apprenions en vivant, en

57 Traitant (en 1951, dans le « Discours de Rome », repris dans (Lacan, 1966 : 237-322) « des rapports dans le sujet de la parole et du langage » (p. 279), Lacan souligne les « paradoxes constituants » (p. 283) et « l'antinomie immanente aux relations de la parole et du langage » (p. 298).

58 Je reprends cette notion lacanienne de « dénaturation » du corps par le symbolique chez C. Soler (2008 : 260) évoquant « les corps qui sont imaginaires dans leur forme, réels en tant que vivants et dénaturés par le symbolique ; cf. aussi « l'effet de langage comme "opération dénaturante" » in Soler (2014 : 21). 
vieillissant, en travaillant, en lisant, nous sommes toujours des chairs où le langage défaille. Nous sommes toujours des anciens enfants, des anciens non-parlants, des bêtes vivipares, des êtres à deux mondes où la langue n'est ni naturelle ni sûre. (Quignard, 2009 : 68-69)

Apparaissent dans ce texte des éléments de ce qui « fait contradiction », de ce qui, pour le sujet, « ne va pas de soi à devenir parlant » et dont la trace demeure au fil de sa pratique langagière de sujet « devenu parlant ».

L'enfant, en naissant, respire, crie, mais - Gargantua excepté - ne parle pas... Cette acquisition fait, certes, partie, dans le développement normal de l'enfant, des diverses conquêtes qui en rythment les premières année : mais elle n'est assimilable à aucune autre. La parole ne survient pas sur le mode où apparaît la marche par exemple (ou, avant elle la reptation, la station debout...) comme manifestation, en temps voulu - chez le petit humain comme chez le petit chien de la fonction, naturelle, de locomotion (même si l'imitation et l'encouragement de l'environnement interviennent dans ce processus). Le langage n'est pas non plus « quelque chose » que rencontre l'enfant dans son exploration du monde qui l'entoure, ni un instrument - hochet, balle, cuillère... - qui lui serait présenté par son entourage, dont il apprendrait à « se servir » et qui, même devenu familier, demeurerait, pour lui, un objet extérieur.

On dit de l'enfant qu'il « entre » dans le langage - qu'il le découvre, se l'approprie... ; on pourrait dire tout autant que le langage entre dans l'enfant, l'investit, se saisit de lui...

De cette opération on peut parler comme d'une greffe $e^{59}$, celle du langage, sur du vivant, qui en fait un sujet humain. Greffer est une opération intervenant dans le développement d'un être vivant, tel un arbre, par exemple, dans le corps incisé duquel l'introduction d'un élément étranger qui va - si la greffe « prend » et n'est pas « rejetée » - se fondre à son porte-greffe et en changer le devenir. Ce que saisit cette métaphore du langage - langue et parole - « enté » sur le petit d'homme par son environnement parlant, c'est, à l'origine même où s'opère la mutation de l'infans en sujet parlant, la contradiction inhérente à l' " être de langage », advenu de ce qu'il incorpore, assimile, fait sien, au plus intime de soi, ce qui, le langage, lui est extérieur, étranger - impliquant que « du dehors » devienne « son dedans ».

Remarque 2 : La nature langagière de l'homme. L'apparente contradiction quant à la «nature » - terme redoutablement polysémique - de l'homme comme " être parlant » qui surgit, par exemple, entre les propositions de Benveniste ( « le langage enseigne la définition de l'homme ») et de Quignard (« la langue n'est ni naturelle, ni [...]»), trouve une issue dans cette conception de l'humain « obtenu » par greffe du langage sur du vivant, par là « dé-naturé ». On peut dire qu'il

59 Cf. Soler (2016), ou Soler (2009 : 5) : « le vivant marqué par le langage ». 
est dans la nature (définition, essence...) de l'homme d'être « dé-naturé », au sens biologique, ou que la « nature humaine » passe par une « dénaturation » - contradiction que Derrida saisit par la métaphore de la « prothèse d'origine » (cf. ci-dessous 2.2).

\subsection{2 "Capture inaugurale » dans le discours de l'autre}

La condition de cette greffe - qui nous ouvre au langage et, à travers lui à la pensée, la conscience, l'échange... et nous fait accéder à notre humanité d'êtrede-langage - c'est ${ }^{60}$ le discours de l'autre, de l'autre en tant qu'il parle autour de nous, et, singulièrement, qu'il nous parle, et qu'au-delà du bain de langage où il nous plonge, il nous implique dans sa pratique : c'est dire que l'origine, la source où nous avons puisé notre langage, c'est le discours de l'autre, premier.

Ainsi, pour l'enfant qui entre - on pourrait dire qui « tombe »- dans le langage, la découverte, vitale, conquérante, que cela constitue, se double - en mode mineur - d'une « capture ». L'appropriation, solidaire, du système de la langue et de l'engagement co-énonciatif de la parole, qui, achevant le processus salvateur de la désadhérence d'avec l'extérieur, ouvre le sujet naissant à la pensée de soi, du monde, et à la relation aux autres, est, indissociablement, pour lui et son dire, " prise » dans les rets de la langue et de son réseau de contraintes et «emprise » des discours environnants ${ }^{61}$.

Avant de revenir ci-dessous sur la plainte qui, longtemps après le premier moment parlant, s'élève, tenace contre cette extériorité dépossédante, rappelons les approches soulignant le caractère fondateur, pour le rapport du sujet au langage, de ce que M. Schneider (1985) caractérise comme « capture inaugurale ». Ainsi, évoquant des manifestations adultes d'un sentiment de « dépossession de sa propre pensée par les mots de l'autre » (telle la hantise, par exemple, de subir ou commettre un plagiat) celui-ci envisage que :

Sans doute est alors révélée, voilée d'un malaise informulable, la reviviscence du temps d'entrée dans la parole, la capture dans les mots de la mère. Cette induction fut traumatique : celle qui nous enseignait la langue nous apprenait d'abord la sienne. (1985 : 295 sq.)

l'acquisition de la langue passant, pour l'enfant, par les discours de ceux qui, $\grave{a}$ leur façon propre, la mettent autour de lui, « en emploi et en action » :

60 Sur la base de «l'équipement génétique qui donne à l'enfant la capacité de parler et lui permet l'appropriation d'une langue «non-innée ». (Jacob, $1981:$ 120).

61 Cf. la métaphore de Lacan (1957), citée ci-dessus 1.1, du « hamac » qui « reçoit » et « emprisonne». 
Il n’y a pas de langue innée. La langue maternelle [...] donnée, reçue [...] n’est pas un outil neutre [...] mais un ensemble de mots investis de désir, de plaisir, d'interdit, d'amour. (ibid. : 285)

Foncièrement, l'enfant qui prend la - « sa » - parole, la reprend « aux lèvres des autres » comme dit Quignard.

Les analyses d'interactions précoces adultes/enfants de 1 à 3 ans, menées, par exemple, par A. Morgenstern ${ }^{62}$ mettent au jour, de façon précise et vivante, la dimension cruciale de l'emprunt - reprise, répétition, imitation... - qui caractérise le « va-et-vient » entre les interactants, à travers lesquels, par « internalisation ${ }^{63}$ des formes reçues de l'autre, se « fabrique » la langue et le sujet.

\section{Remarque 3 : La reprise initiale : ni « perroquet » ni RDA.}

(1) De façon précieuse, A. Morgenstern (2007) souligne que « la reprise de formes entendues dans le discours adulte » menant à une acquisition, c'est-à-dire à leur appropriation, ne se confond pas avec l'écholalie, ou une imitation relevant « d'un simple automatisme passif » : la reprise « énoncée » passe par une « forme de sélection qui traduit de l'intentionnalité ».

C'est lorsque la « greffe » n'a pas - ou mal - pris que surgit une parole « de perroquet ». Dans le même article l'auteur oppose à la vraie reprise créatrice, le caractère « échoöque » de la parole d'enfants présentant des troubles du langage ${ }^{64}$, et émettant d'une voix « inexpressive », " sur un schéma mélodique indéfiniment répété », un " patchwork » de formulations entendues. (2) Sélectionnés, intentionnels, les reprises, emprunts par lesquels émerge le langage, n’apparaissent certes pas explicitement redoublés comme tels au plan métalangagier par des « comme tu dis » par exemple. Pour autant, notamment, dans l'observation ${ }^{65}$ des « renversements pronominaux » où l'enfant (dans sa troisième année) parle de lui en tu (« bravo tu marches !! » d'un enfant qui parvient à faire quelques pas avec les souliers de son père), l'auteur, évoquant pertinemment le fait que l'enfant « exprime sa propre fierté en empruntant le rôle de "congratulateur" habituel de sa mère », parle d'une « forme de dédoublement de soi », de dimension « citationnelle» (et non pas simplement « échoïque »).

Ne faut-il pas considérer que l'émergence du langage se double, précocement, d'une conscience du langage ? Les reprises « citationnelles " peuvent-elles être envisagées comme de la « proto-RDA », des ébauches de ce qui deviendra la RDA « dite » ${ }^{66}$ ?

$62 C f$. les discours « repris, empruntés, habités » des titres de A. Morgenstern (2007, 2012).

63 Terme de Vygotsky, cité en conclusion, dans Morgenstern (2012).

$64 C f$. les remarques de C. Soler (2008: 80) sur certaines thérapies d'enfants psychotiques dont les paroles « en progrès » restent cependant du côté du « perroquet » et de « l'écho » du dire des autres (cf. ci-dessous 2.3.2, p. 521).

65 Morgenstern (2007, section 3).

66 Cette question affleure dans l'analyse de A. Morgenstern (2007, section 1) à propos d'un enfant de 21 mois « allant de pièce en pièce [...] en babillant et en pointant tout ce qui se trouvait sur son passage », dans le commentaire : " On peut se demander si le pointage ne serait pas du gestuel "rapporté”. [...]. [...] il reprendrait alors la "mise en geste" de l’adulte. » 


\subsubsection{Mère « porte-parole ", infans « parlé "}

Que ce soit depuis la parole de l'autre - dès lors, condition et source - qu'émerge la parole de soi par reprise, établit donc, en même temps, l'emprise de la première sur la seconde.

En deçà de ce plan où le rapport intérieur/extérieur s'établit réciproquement entre l'un et l'autre, en appropriation/capture, apparaît un niveau plus radical de captation, exploré notamment par P. Aulagnier ${ }^{67}$, au-delà et du fait que :

Précédant de loin la naissance du sujet, lui préexiste un discours le concernant (ibid. : 131)

comme le lieu d'une « violence primaire » exercée sur le non-parlant :

La parole maternelle déverse un flux porteur et créateur de sens qui anticipe de loin sur la capacité de l'infans d'en reconnaître la signification et de la reprendre à son compte. (1975: 36)

En deçà de (ou incluse dans) la parole adressée à l'enfant - celle par laquelle on lui parle - il faut faire la part de ce qui relève d'une parole qui le parle, qui parle de lui, qui parle pour lui, à sa place, qui le «met en mots », le définissant et « traduisant » (« interprétant ») ses besoins et émotions... ${ }^{68} \mathrm{P}$. Aulagnier évoque ainsi :

La violence primaire qu'exerce l'effet d'anticipation du discours maternel [qui] se manifeste essentiellement par cette offre de signification qui a comme résultat de lui faire émettre une réponse qu'elle formule en lieu et place de l'infans (ibid. : 41)

conférant au discours maternel une fonction de « porte-parole ». Avant de citer le mode - double - sur lequel elle en déploie, théoriquement, la métaphore, on peut, à un plan élémentaire, noter que l'expression « discours porte-parole » fait jouer le double sens d'une parole donnée - transmise à quelqu'un comme l'est la flamme apportée par le coureur olympique - et substituée à une parole qui n'a pas la possibilité de se dire :

Ce terme définit une fonction dévolue au discours de la mère dans la structuration de la psyché : porte-parole au sens littéral du terme puisque c'est à sa voix que l'infans doit, dès sa venue au monde, d'être porté par un discours qui tour à tour commente, prédit, berce l'ensemble de ses manifestations, mais porte-parole aussi au sens de délégué, de représentant d'un ordre extérieur dont ce discours énonce à l'infans les lois et les exigences. (ibid. :130)

67 Aulagnier P., 1975, La violence de l'interprétation.

68 Ceci pour s'en tenir à une simplification extrême d'une pensée riche et difficile. 
Contre le sentiment - normal - du parlant habitué à " se servir » du langage comme d'un instrument dont l'espèce humaine aurait le privilège de disposer, les « ratés » - psychotisants - de l'entrée dans le langage témoignent ${ }^{69}$, dans la souffrance et la catastrophe, de cette " hétéronomie » du langage qui, " greffé » sur le vivant, peut « ne pas prendre »- pas vraiment, ou pas du tout ${ }^{70}$.

L'appropriation réussie qui nous fait vraiment " parlant » ${ }^{71}$, c'est-à-dire nous permet de nous impliquer, confier, inscrire dans le langage - et parfois nous y consacrer par l'écriture - ne va pas cependant sans une interminable plainte qui, même si elle emprunte volontiers la forme de récriminations à l'encontre d'un « mauvais outil ${ }^{72}$ résonne - parfois explicitement - comme un écho de ce qu'il y eut de " perte » à entrer dans le langage ${ }^{73}$, comme une douleur, plus ou moins vive, de « cicatrice » au lieu de la greffe initiale.

\subsection{Un sujet ex-centré par l’Autre du langage : variations théoriques}

Remarque 4 : L'Autre du langage vs les autres discours. On a déjà, chap. 10.3.2, évoqué l'imaginaire comme lieu de la relation à autrui, un autre semblable à soi. A. Vanier (1998:40) résume ainsi le mode sur lequel l'Autre s'oppose à l'autre :

La relation à l'autre est fondamentale pour Freud dans la constitution du sujet. Lacan distingue l'autre, le semblable, le partenaire imaginaire, celui avec qui se jouent les phénomènes d'identification, de l'Autre, place essentielle de la structure du symbolique, qui se confond à la limite avec le langage et intervient au-delà de l'autre dans toute relation humaine (idt) ;

ce que J.-P. Lebrun (2015 : 56) formule à son tour ainsi :

Lacan distingue donc deux " autres " : un premier qu'il écrit avec une minuscule, l'autre qui est le partenaire, et un second qu'il écrit avec une majuscule : l'Autre qui, au-delà du

69 C. Soler (2016), à propos de la caractérisation par Lacan du psychotique comme « un martyr du langage », rappelle que le martyr est celui qui souffre et témoigne.

70 Je renvoie à Manier (1995) et Soler (2008); et voir ci-dessous 2.3.2.

71 « Parlant » que A. Manier (1995 : 45) oppose à l'enfant psychotique, qui sera « au mieux phonant », « faute qu'on l'ait parlé, qu'on lui ait donné la parole ».

72 Pour reprendre le titre du livre de P. Henry (1977).

73 " Perte de l'état premier ", " extase fusionnelle », " densité de la chose même " (Manier, ibid. : 119), si présente chez Quignard ( $c f$. ci-dessus, ou encore "l'ancien silence » " qui fait le fond de l'âme » in 2012 : 164-168), et qui est « le prix à payer en échange de la capacité de représentation » (Manier, ibid. : 122). 
partenaire, désigne la scène du langage qui, antérieure et extérieure au sujet, le détermine néanmoins radicalement. [...] ce grand Autre [n'étant] accessible à quiconque qu'au travers de ceux- les parents, et même plus directement la mère - qui lui ont prêté chair.

Pour s'être approprié le langage, le sujet, devenu parlant, n'en a pas fini - n'en aura jamais fini - avec, dans sa parole « propre », la contestation qu'y porte son ancrage nécessairement extérieur : la contradiction est indépassable dans une parole dont l'énonciateur se pense - et a besoin de se penser - « source » pour pouvoir la vivre comme sienne, alors que lui préexiste nécessairement le langage dont elle émane, sous la loi de la langue et l'emprise des discours.

À cette contradiction, inhérente à la parole, le sujet aura toujours à répondre, c'est-à-dire à trouver l'équilibre singulier, instable, mouvant, jamais établi une fois pour toutes, selon lequel il pose sa parole dans le langage qui l'en dépossède dans le moment où il la lui donne. Et, pour un sujet « effet de langage » - c'està-dire qui est sujet d'être parlant -, cet équilibre, toujours à réassurer, de façon vivante, $\mathrm{du}$ « propre » de sa parole dans l'Autre du langage, est partie prenante de la constante " refabrication $~^{74}$ de lui-même, comme une des dimensions par lesquelles celle-ci s'opère.

Un concert de voix - linguistes, philosophes, psychanalystes, écrivains ${ }^{75}$ s'élève pour dire le « prix à payer » de l'accès au langage et, par lui, à la subjectivité, à la pensée, à l'échange... Explorant ce qu'il en est de « perte » au don du langage, à ce que la parole « propre » doive en passer par des mots « pas à soi », ces variations langagières sur le langage incarnent, dans leur tonalité disphorique dominante, la contradiction inhérente à celui-ci : s'en remettre au langage pour se plaindre de lui...

Quels que soient les modes - frustration, aliénation, dépersonnalisation, dépossession, expropriation, etc. - sur lesquels se trouve vécu et nommé le

$74 C f$. Henry (2012) : « ce sujet, ce parlêtre, ce sujet fait de langage [...] ne cesse pas d'être en " refabrication », en recomposition, en recollement. [...] cette refabrication [...] s'inscrit dans un contexte, un environnement, des situations de dialogue... dans l'histoire individuelle, dans l'histoire tout court... Le sujet parle, écoute, et parce qu'il parle et écoute, il est constitué et modifié sans cesse par son propre discours et parce qu'il s'approprie le discours qu'il reçoit ».

75 Les deux «pratiques de langage » - travail et aventure - que sont la psychanalyse et l'écriture littéraire, certes foncièrement différentes, mettent, toutes deux, à vif la contradiction entre la singularité subjective et le « commun » du langage, présentant, comme autant de réponses à cette contradiction, des modes propres, parfois extrêmes de « se poser » dans le langage. Le champ est immense, tant au plan de la diversité des « observables »- paroles de sujets « en analyse » ou écritures littéraires - que des réflexions à leur sujet ; et il n'est pas question, ici, au-delà de brèves évocations, dans ce qui suit, de quelques exemples, d'en rendre compte. 
négatif de l'expérience langagière, il importe de ne pas rabattre celle-ci sur les rencontres qu'un sujet parlant peut faire de l'emprise sur son dire d'un autre discours (spécifique) distinct : c'est le langage lui-même, "voix de personne », qui est ici à l'œuvre, dépossédant le sujet " par des mots sans auteur » ${ }^{76}$. Derrière les effets, potentiellement frustrants ou aliénants, des émergences « dialogisantes » imprévues dans le discours en train de se faire, depuis le milieu du déjà-dit des autres discours, s'opère, pour le sujet, qui en devient " parlant », une « ex-centration » dans l'Autre, ou l'ailleurs du langage lui-même - avec lequel il n'est pas de rapport « de dialogue »...

Remarque 5 : La « chose perdue ». La « perte » inhérente à l'accès au langage ne relève pas seulement de la « capture » dans un ailleurs, elle loge aussi dans l'irrémédiable écart que les signes instaurent entre le sujet et le monde : l'accès à la représentation par le symbolique libère - et protège - le sujet du mortifère « faire un " avec la chose, et, par là même, ouvre sur la nostalgie, la souffrance de la " chose perdue ${ }^{77}$. Évoquée dans Moi, monde, mots par F. Gantheret (1996:17) :

[...] pathos humain, souffrance et passion d'être jeté dans le monde, de n'y être pas « naturel », de devoir le parler, à tout le moins le mettre en signes. Passion d'interroger fébrilement ces signes. Et souffrance d'éprouver qu'ils ne sont jamais, contre notre espoir, la chose même que nous voulons rejoindre.

cette souffrance d'une distance, d'un intervalle impossible à réduire a souvent été placée au principe même de la littérature comme pratique de cet écart dont, dit Barthes (1978 : 22) « les humains ne prennent pas leur parti »; mais, si profonde soit-elle, la mélancolie de la chose perdue n'entre pas en résonance avec notre objet, du statut dans le dire de la représentation du discours autre, aussi directement et spécifiquement que l'expérience du « pas à soi » du langage.

\section{La singularité passée par la loi de la langue}

De ce que « la langue est un système commun à tous ", alors que « les configurations de la parole sont chaque fois uniques », Benveniste (1966 : 78) conclut : " Il y a antinomie chez le sujet entre le discours et la langue ».

Aucun dire n'advient en dehors de la langue reçue " en partage ", loi $^{78}$ impersonnelle, commune, sous les fourches caudines de laquelle - système de règles, de limites - il doit passer pour prendre corps. De ce qu'il en coûte de renoncer à « l'impossible - parler une langue qui ne serait qu'à moi et que pour-

76 Cf. Schneider (1985: 230) et (2010:56).

77 Cf. Authier-Revuz (1995/2012, chap. 9).

78 «Loi » qui, souligne Derrida (1996/2016:69) « demeure nécessairement, ainsi le veut au fond l'essence de toute loi, hétéronome » (idt). 
tant chacun pourrait entendre ${ }^{79}-$, les réflexions suivantes se font, parmi bien d'autres, l'écho, à travers leur visée propre - celle, par exemple, linguistique, de G. Siouffi :

Cette aliénation qui a comme horizon le caractère extérieur des mots, leur matérialité, le fait qu'aucun des mots que j'emploie en tant que sujet n'est mien [...] peut très naturellement être perçue comme une aliénation négative, destructive de l'expression authentique du sujet, « impersonnalisante » [...]. La valeur d'échange du langage, son caractère arbitraire et conventionnel, est (idt) profondément une aliénation. (Siouffi, 2001 : 191, 195)

- celle, psychanalytique de G. Pommier :

Le fait de parler, de s'approprier la langue, impose une mortification au sujet. Celui-ci emploie des mots qui ne lui appartiennent pas, et il se soumet à leur logique, qui lui échappe de toute part. [...] l'agent de cette mortification est le père mythique qui a créé la langue. (Pommier, 1999 : 122)

- ou celle, philosophique de M. Merleau-Ponty, posant que subjectivation (par le langage) implique dépersonnalisation :

Il n'y a de parole (et finalement de personnalité) que pour un « je » qui porte en lui ce germe de dépersonnalisation. (Merleau-Ponty, 1952 : 29, cité par Siouffi, 2001)

\section{Le Dire, un RE-dire obligé}

Au rappel, par Saussure, au plan de la langue, de l'avant de « ce qu'on a dit » sur quoi se fonde ce que «nous disons»:

Pourquoi disons-nous homme, chien ? Parce qu'on a dit avant nous homme, chien. La justification est dans le temps. (1968, tome $1: 165)$

répondent, au plan du discours et de l'énonciation, évoqués au chapitre 10, le mythe bakhtinien du solitaire Adam et de sa parole toute neuve... émergeant dans un monde innommé, comme le "ça parle ailleurs, avant et indépendamment » de M. Pêcheux. Objet de désolation, d'exaspération, de hantise même au fil des siècles, de la part de tant de penseurs ou d'écrivains ${ }^{80}$, cette dimension de fon-

79 Pontalis (1986: 28).

80 Pensons à Flaubert « vouant » son écriture à faire texte de cette inévitable dépendance à la « Bêtise » (cf. chap. 14), ou aux stratégies défensives de Nietzsche face à la menace de la grégarité, 
cière dépendance du dire à ce qui langagièrement le précède traverse fortement la pensée contemporaine, sensible à l'emprise des discours.

Ainsi, dans la conception foucaldienne d'un « Ordre du Discours », déterminant le dire, l'excentration dans laquelle celui-ci se produit, depuis une " pensée du dehors », se fait-elle, en même temps, depuis l'avant, dans

[...] le ruissellement et la détresse d'un langage qui a toujours déjà commencé (La pensée du dehors, in 1966 : 523)

et on note, chez J.-F. Lyotard, l'insistance - soulignée par le retour du nous - sur la commune expérience pour le sujet parlant de la préséance du déjà-dit sur son dire :

[...] nous sommes toujours sous le coup de quelque récit, on nous a toujours dit quelque chose et nous avons toujours été déjà dits (1977 : 47)

Pour Derrida, cet avant, par rapport auquel la parole, dès son commencement - et à jamais - sera « itérative " pour un sujet débouté comme source ou origine de ce qu'il dit, est ce qui fait « une aliénation essentielle dans la langue - qui est toujours de l'autre ${ }^{81}$ :

Dès que je parle les mots ne m’appartiennent plus, ils sont originellement répétés. [...] Ce qu'on a appelé le sujet parlant n'est plus celui-là seul qui parle. Il se découvre dans une irréductible secondarité [...], origine toujours dérobée à partir d'un champ organisé de la parole dans lequel il cherche en vain une place toujours manquante. (La parole soufflée, in $1967: 264 s q$.)

La langue, " prothèse d'origine ", est foncièrement " étrangère ${ }^{82}$, et la parole qui - d'être répétée - relève de la « citation ${ }^{83}$, passe de surcroît par le mou-

ou de Barthes face à celle de la doxa ( $c f$. ci-dessous 2.3.5, p. 540). Je renvoie au riche parcours que M. Schneider (1985) consacre à ceux qui s'éprouvent, au cœur de leur dire, comme « Voleurs de mots".

81 Cf. (1996/2016 : 113-114), ou encore :

une sorte d'aliénation originaire qui institue toute langue en langue de l'autre : l'impossible propriété d'une langue (ibid. : 121).

82 Cf. le « je n'ai qu'une langue et (mais, or,...) ce n'est pas la mienne », leitmotiv de « Le Monolinguisme de l'autre ou la prothèse d'origine » (1996/2016).

83 On retrouve ici le sens IV de « citation », ou « citationnalité », évoqué dans l'appendice au chapitre 9. On revient, chap. 14.1.3.4 3 p. 565 sq., sur la nécessité de ne pas écraser la différence entre la citation-RDA et la « citation » comme inhérente au langage. 
vement d'une « importation ${ }^{84} \mathrm{du}$ langage de l'autre » ou celui - « sans langue originaire, sans langue de départ »- d'une " traduction ${ }^{85}$ dans ce langage de l'autre.

L'excentration de la parole par l'ailleurs-avant qui, jouant en elle, la déporte hors d'elle-même, est pensée chez Derrida en terme d'expropriation (ou d' « exappropriation »), et l'énonciation ne sera " jamais de part en part présente à ellemême et à son contenu » (1972, repris dans 1990 : 46) de ce que "L'itération qui la structure a priori y introduit une déhiscence et une brisure essentielles » (ibid. : 195) ${ }^{86}$.

\section{Des mots " pas à soi » à l'" exil » dans les mots}

À travers la variation ci-dessus sur le « pas à soi » du langage - signes « communs » qu'impose la langue, mots « de l'autre » qu'il faut « importer » - le thème de l'expérience du langage ${ }^{87}$ comme dépossession apparaît comme récurrent.

À parcourir le domaine du plagiat - de son sens strict (plagier/être plagié) au sens étendu de ce qu'implique un langage, qu'on ne peut que s' « approprier » l'ouvrage Voleurs de mots de M. Schneider (1985) est un voyage en « inappartenance foncière du langage » :

Le propre des mots est d'être impropre ; leur destin d'être volés, ou de vous voler, ne vous dérobent-ils pas à vous-mêmes, déposant en vous des pensées insues, des réminiscences involontaires ? [...] Entre mes mots et moi, une dépossession : quelqu'un qui me les donne, me les prend $[. .$.$] ( 4^{\mathrm{e}}$ de couverture)

Et l'image est constante chez Bakhtine (cf. chap. 10.1.2, p. 389) du locuteur contraint, à jamais dépourvu qu'il est de mots « à lui », d'aller prendre « ses » mots en « territoire » étranger, et s'efforcer de se les « approprier».

$84 C f$. : « L'importation commence dès le premier mot ; on apprend à parler en important mot par mot» (1996/2016).

85 La double distance qu'opèrent « citation » et « traduction » (ou « importation ») est ce que souligne A. Sevenant (1997) en achevant Importer en Philosophie, l'essai qu'elle consacre à Derrida, par cette phrase : « L'expression citer dans une autre langue (idt) marque et remarque ainsi le caractère disjoint de toute langue » (p. 157).

86 Posé dans l'essai Signature, événement, contexte qui clôt Derrida (1972), le fait que le statut constitutivement itératif de la parole affecte celle-ci d'une " absence à elle-même », n'annule pas la réalité de l'intentionnalité énonciative qui - objet d'une vive contoverse avec Searle - est réaffirmée, en réponse polémique à celui-ci, en 1990, dans Limited Inc.

$87 \mathrm{Au}$-delà du temps de « l'acquisition par l'enfant de la langue du groupe qui le précède [...], qu'il peine à faire sienne. » (Quignard, $2012: 17$ ) 
Au-delà, ce qui se dit à travers la métaphore prégnante de l'exil du sujet dans le langage, c'est - plus radicalement que d'avoir, depuis un « chez soi » subjectif, à en passer par des mots d'ailleurs - la perte de tout « chez soi » que signe, pour le sujet, son entrée dans le langage.

Ainsi de la « radicale extériorité de l'humain par rapport au langage » qu'il rappelle, O. Grignon en vient-il au

rapport forcément étranger, [...] d'exil fondamental des hommes par rapport au langage. (2017: 91-92)

cependant que E. Lemoine-Luccioni donne à ce chez-soi « perdu » le visage d'une « maison » imaginairement originaire :

Il a giclé un jour hors de son gîte pour naître [...]. Mais il tombe dans le tout fait du langage et le voilà perdu dans la maison commune; dans l'unheimlich en tout cas, car il ne retrouve jamais sa maison, qu’il n’a pas eue. (1983: 91$)^{88}$

et que J.-B. Pontalis - dont le beau livre de souvenirs, L'Amour des commencements, suit les formes prises par ce qui, à ses yeux, « commande » sa vie : «l'amour et la haine du langage » - évoque

[ce qui] fut pour [lui] le premier maléfice du langage : en lui je ne pouvais être effectivement qu'immigré, déplacé, sans que cet exil forcé me donne pour autant la nostalgie d'une terre natale. Le maniement du langage me ferait perdre jusqu'au pouvoir de me représenter ce que j’avais perdu ! (1986: 8$)$.

Et de même, pour Derrida, si la langue maternelle est « habitable », c'est dans l'exil endeuillé d'un vrai « chez soi » :

La langue dite maternelle n'est jamais purement naturelle, ni propre, ni habitable [...] Il n'y a pas d'habitat possible sans la différence de cet exil et de cette nostalgie. (1996/2016:112)

et le « grief»

[...] quasi-originaire, puisqu'il ne déplore même pas une perte : [...] dans un tel grief, on prend ainsi, à demeure, le deuil de ce qu'on a jamais eu (ibid. : 61)

88 Das Unheimliche est le titre d'un texte de Freud, traduit par « l'inquiétante étrangeté », étymologiquement formé sur « heim » : à la maison. 


\section{Le dire dispersé en voix multiples}

De cet ailleurs-avant d'où procède le dire du sujet, c'est aussi la pluralité des voix qui y « parlent » qui est soulignée - pluralité dont le dire est fait et où se défait le caractère « un » que l'on prête au conscient ${ }^{89}$.

Ainsi Milner (2011 : §5), rappelant que

Dès qu'il n'est plus infans, le sujet aura à s'arranger de la pluralité parlante. [...] l'être parlant est toujours déjà plusieurs en tant qu'il est parlant (p. 15),

\section{évoque-t-il}

la foule intérieure qui persiste au plus intime de chacun et dont la langue se fait le portevoix (p. 16).

Et centrale est, chez Deleuze, la pensée d'une « désindividuation » : la position radicale selon laquelle

Il n’y a pas d'énonciation individuelle [mais un] agencement collectif impersonnel [...] qui va déterminer comme sa conséquence les procès relatifs de subjectivation, les assignations d'individualité (Deleuze et al., 1980 : 101)

se $\mathrm{dit}^{90}$ (dans Mille Plateaux, par exemple) à travers la métaphore récurrente du « discours indirect » ou du « discours indirect libre » comme principe même du fonctionnement du « discours direct $»^{91}$ qui, jamais « premier », fonctionne à l'ouï-dire :

Le discours indirect est la présence d'un énoncé rapporté dans l'énoncé rapporteur, la présence du mot d'ordre dans le mot. C'est le langage tout entier qui est discours indirect. Loin que le discours indirect suppose un discours direct, c'est celui-ci qui s'extrait de celui-là [...]. (1980 : 106).

89 Cf. Milner (2011 : 14) « Puisque la tradition philosophique a donné le nom de conscience au principe d'unicité, on comprend qu'à raturer ce nom par le nom d'inconscient, on précise l'insistance, au plus secret de l'être parlant, de son être plusieurs » (idt).

90 Conjointement à la conception du fonctionnement, socialement chargé de discours, du mot comme « mot d'ordre » imposant ses « présupposés implicites » (1980 : 97-100).

91 « discours direct » étant ici pris (comme souvent dans le discours philosophique) au sens de " en direct », non rapporté, voir chap. 2.1. 
Cette métaphore qui « transporte » DI, ou DIL, de leur valeur linguistique de formes localisables de $"$ discours rapporté ${ }^{92}$ vers un statut de tout discours comme relevant du « DI », « DIL »..., procède du même « mouvement » que celui, rencontré ci-dessus, menant du fait de la citation en discours au discours comme citation. Mais, dans l'ensemble des traitements du dire comme foncièrement traversé par l'autre qui le précède, s'impose, singulière, chez Deleuze, la tonalité joyeuse du constat de la défaite $d u$ « $U N$ » dans une énonciation « originairement plurielle » - tonalité qui (accompagnée d'un lexique où l'autre, le différent, l'inégal... consonnent avec le vivant et l'intense) s'entend dans le leitmotiv ${ }^{93} \mathrm{du}$

[a] tant d'êtres et de choses pensent en nous...,

[b] toutes les voix présentes dans une voix, les éclats de jeune fille dans un monologue de Charlus,

[c] toujours une voix dans une autre voix...,

[d] toujours un autre souffle dans le mien, une autre pensée dans la mienne,

et s'explicite dans le « joyeux message » que constitue la reconnaissance d'un « moi dissous » qui s’ouvre à une multiplicité d'autres qui le « font » :

Il ne s'agit pas des influences que nous subissons, mais des insufflations, des fluctuations que nous sommes [idt], avec lesquelles nous nous confondons. Que tout soit si « compliqué », que Je sois un autre, que quelque chose d'autre pense en nous [...] dans une violence qui est celle du langage, c'est là le joyeux message. (Deleuze, 1969 : 346)

\subsection{Extériorité du langage et parole propre}

Appréhender l'antinomie fondatrice du langage c'est, immanquablement - loin du tranquille rapport du sujet à un « instrument » reçu en partage - voir la parole surgir comme question. Dans un chapitre intitulé « Ce que parler implique », J.-P. Lebrun illustre, en une page, ce mouvement de constat d'une contradiction :

92 Formes de RDA que Deleuze évoque comme « insertion d'énoncé “différemment individués” ou "emboîtements de sujets d'énonciation divers", à penser comme phénomène second, de surface si l'on veut, par rapport à l' "agencement collectif " qui sous-tend et détermine "les procès relatifs de subjectivation, les assignations d'individualité et leurs distributions mouvantes dans le discours" ». (Deleuze, $1980: 101)$.

93 J'emprunte le terme à F. Zourabichvili (1994: 125), comme aussi les citations qui suivent [a], [c] de Deleuze (1969 : 347) et (1985 : 218), [b], [d] de Deleuze et al. (1980 : 101, 346). 
ce que j'ai de plus singulier, mes mots, qui est aussi ce que j'ai de plus intime [...] pourtant n'est jamais construit que dans le matériau de l'Autre (2015: 57),

qui se fait aussitôt énigme à résoudre :

Mais alors comment le « singulier » d'un sujet peut-il se constituer à partir du « commun » qui lui vient entièrement de l'Autre ? [.] À quelles conditions [...] Pourquoi ne reste-t-il pas complètement aliéné dans l'Autre ? Comment lui est-il possible [...] ? Pourquoi ne reste-t-il pas noyé dans l'Autre, englué, incapable de distinguer ce qui est de lui et ce qui est de l'Autre? (ibid.)

Ainsi, pour «normal » qu'il soit, pour le petit d'homme de devenir parlant, il ne faut pas ignorer la force de l'opération inaugurale par laquelle l'infans accède à sa parole à partir du langage reçu de l'autre, ni la tension, jamais levée, dans laquelle tout dire aura à trouver son (dés)équilibre entre extériorité du langage et parole énoncée comme sienne (2.3.1).

Sur ce qui se joue, crucialement, dans l'opération première d'entrée dans le langage, comme de sa nécessaire réassurance par le sujet parlant, de graves troubles langagiers témoignent "par défaut », apportant un éclairage aigu sur l'ordinaire de la parole «normale » (2.3.2).

\subsubsection{L'appropriation fondatrice et son renouvellement}

En termes d'aliénation, dépossession, exil, déhiscence... on a dit ce qu'il y a de « perte » - d'un état primitif de fusion... - à en passer par l'Autre de la langue et des discours : c'est via cette « perte » que pourtant - ou plutôt « justement »l'enfant accède à la parole car le langage " greffé » qui, de toute son extériorité, excentre le sujet, est ce qui, par là même, l'ouvre sur l'ailleurs et qui, reçu de l'extérieur des autres qui lui parlent, lui donne prise sur un monde qu'il peut nommer et accès à des autres auxquels il peut s'adresser ${ }^{94}$.

C’est comme une « deuxième naissance » qu'A. Manier (1995 : 51) célèbre :

[ce] moment d'excentration d'une étonnante audace et fécondité qui a consisté à pouvoir énoncer par un signifiant une unité de pensée en accord avec l'autre.

94 C'est l'oubli de ce que l'aliénation comporte d'ouverture que dénonce P. Henry (1977 : 162) : « [...] nos corps sont pris dans le langage avant toute cogitation. N'y voir que le fondement d'une aliénation quasi-existencielle, c'est oublier que le langage est aussi ce qui rend possible une appropriation du réel [...]. » 
L'accès au signe partagé avec l'autre marque la fin - deuil et libération - de « l'immédiateté de la relation à l'objet ${ }^{95}$, ouvrant le sujet, désormais séparé de la « chose », à l'espace de la pensée et au jeu de l'intersubjectivité.

Le « miracle » - si commun soit-il - des premiers mots est celui d'une appropriation : l'enfant « prend » $s a$ parole à partir de celle qu'on lui adresse, se faisant $d u$ « propre » avec du non-propre. La conversion qu'accomplit l'enfant, de l'extériorité du langage dans une parole de soi, dépend - tout au long du chemin qui le mène du cri à l'appel et à la parole - de l'expérience qu'il fait de la parole à lui adressée et de la subjectivité qui anime celle-ci : c'est dans cette énonciation de l'autre qu'il trouve, plus ou moins aisément, plus ou moins profondément, l'élan - désir et confiance - qui le porte à " s'expatrier » dans des mots d'ailleurs ${ }^{96}$; aucun dispositif technique, si sophistiqué soit-il, ne pourrait susciter la réponse d'une première parole.

Caractérisant ce moment crucial de l'entrée dans la parole comme « constitutif de la distinction extérieur-intérieur » E. Porge (2012 : 90) souligne que si cette distinction est :

corrélative de toute identification, [elle est] corrélative aussi d'un retournement où il y a un extérieur de l'intérieur.

Décisive et émouvante, l'appropriation initiale de la première parole ne « règle » pas la contradiction inhérente à l'extériorité interne au dire, n'annule ni n'aplanit la tension entre le fait réel que les mots - relevant de la langue commune et chargés de leur histoire discursive - ne sont pas à soi, et l'impérieuse nécessité subjective d'une parole énoncée comme propre. La structure topologique de l'extériorité interne est le réel que toute parole a à affronter, expérimenter, vivre. Ce que M. Schneider analyse de cette contradiction, à propos de l'œuvre littéraire, on peut - sans pour autant les confondre - le dire pour la parole « ordinaire » :

On ne peut pas dire : mes mots à moi » sans s'illusionner sur la nature du langage et des mots qui ne sont pas plus à moi qu'à personne. Par contre, je peux dire : mon œuvre [...]. La propriété ne concerne que l'œuvre et non ce dont elle est faite. La difficulté logique se pré-

95 Il est facile, du fait de sa violence, de ne faire résonner la formule « le mot est le meurtre de la chose » que sur le versant de la perte, ignorant, dans la contradiction inhérente au langage, le versant, strictement solidaire, de pouvoir et de protection que confère au sujet un accès à l'objet, distancié et médié par un système de représentation partagé. Sur l'enveloppe langagière protectrice qui dramatiquement fait défaut au psychotique, $c f$. par exemple Manier (1995:111-112).

96 Et, bientôt, à « apprendre à dire "je" : apprendre que "je" existe à travers la plus sociale des institutions, la moins individuelle : la langue » (P. Lepape, Le Monde des livres, 30 janvier 1998), cité in Morizot (2001 : 5). 
sente donc ainsi : comment le texte, simple assemblage de mots, choses viles et communes, peut-il être dit mien, légitime, défendu comme propre ? (1985 : 321)

On peut pour toute parole, on l'a dit, se poser la question de ce «comment» : comment une parole peut-elle s'énoncer comme propre à partir des mots partagés de la langue et du déjà-dit ? Évoquant ainsi l'émergence du propre depuis l'étranger :

Le propre n'est jamais que ce que je fais mien de l'étranger, ce que je m'approprie, par des voies toujours singulières. (1985: 309)

M. Schneider pointe un élément crucial : le caractère singulier des voies de l'appropriation. L'issue à la contradiction - «miracle » inaperçu au cœur de chaque dire - est ce qu'accomplit l'énonciation comme acte d'appropriation singulier - le temps du dire - de ce qui vient de l'autre. Énoncer, c'est se faire source à partir du flux qui nous traverse. Chaque parole renouvelle - et avec elle l'alliance initiale de l'entrée dans le langage - la conversion « appropriante » de l'inappartenance réelle des mots dans l'affirmation subjective de leur énonciation singulière ; et dans cette expérience, selon le mode où le sujet s'y engage, se joue le sentiment de son identité.

Ainsi, vivre pour un sujet parlant, c'est-à-dire dans le langage, est-ce d'emblée et pour toujours, répondre à la contradiction d'un dedans fait du dehors en y « posant » $s a$ parole, dans un (dés-)équilibre singulier, mouvant, incertain parfois, ou difficile, et que des troubles graves peuvent, tragiquement, mettre hors d'atteinte.

\section{Remarque 6 : Appropriation singulière et « originalité ».}

(a) Reconnaître la singularité de l'acte d'appropriation par lequel, dans le moindre dire, le sujet se fait source de ce qu'il énonce via les mots qu'il a reçus, ne touche nullement à la question de l'« originalité » de la parole produite. Faire sien ce qui vient de l'autre c'est ce qu'accomplit toute parole : autant celle qui puise, joyeusement insouciante au déjà-dit offert, que celle en proie à un vrai tourment de devoir en passer par lui - Flaubert, Nietzsche, Barthes... parmi tant d'autres que persécute l' « usure » des mots - ; autant dans l'affirmation souveraine de voix « inouïes » - Rabelais, Hugo, Rimbaud... - que dans l'effacement de paroles comme étouffées qui se coulent étroitement dans les moules du déjà-dit ou en empruntent les chemins les plus balisés, telles, par exemple, celles des marionnettes désindividualisées que mettent en scène N. Sarraute, Pinget ou Vinaver ${ }^{97}$.

97 Précisément étudié sous le titre « La Parole empruntée [...] : théâtres du dialogisme » par E. Eigenmann (1996) 
Aussi usée, répétitive, stéréotypée... que soit une parole, elle est acte unique, singulier, par lequel un sujet prête vie, corps, souffle, trace aux mots communs disponibles qu'il « habite » et « fait sien ».

(b) N'oublions pas, évoqué ci-dessus, chap. 5.1.2, p. 150, ce fait essentiel que le plus « commun » des énoncés est toujours, dans le contexte singulier de son énonciation - contrairement à la phrase à laquelle il prête vie - un acte unique, non répétable.

(c) Rappelons, enfin, cet irréductible espace de singularité que recèle tout énoncé, si « commun » soit-il, en ce que sous la surface des mots qu'énonce le sujet, s'ouvre une autre altérité que celle de l'interdiscours ou du dialogisme : celle, inépuisable envers de la langue, de lalangue ${ }^{98}$ qui, « en toute langue [est] le registre qui la voue à l'équivoque », par qui toute langue est aussi « mode singulier de faire équivoque ${ }^{99}$, et dont le jeu imprévisible accompagne l'énoncé le plus convenu. Ainsi, faisant alliance avec la langue chargée de discours lorsqu'il y élit en les « faisant sien » les mots de son énoncé, le sujet fait-il en même temps - et ne peut pas ne pas le faire - alliance avec lalangue où de façon sauvage, radicalement singulière et immaitrisable ${ }^{100}$ s'inscrit (largement à son insu) son désir inconscient - le sujet habitant ainsi doublement ce qu'il énonce sur les deux portées de son dire ${ }^{101}$. Par la « portée du dessous » qui y court, le dire le plus prévisible et le plus commun s'énonce - fut-ce, pour son énonciateur, non seulement à son insu mais à son corps défendant - comme parole singulière.

98 Pour une présentation schématique de ce concept lacanien, $c f$. Authier-Revuz (1995/2012: 655 $s q$.$) ; réflexion éclairante in Soler (2009)$

99 Milner (1978 : 22) et (ibid. : 104) : par rapport à la langue réseau descriptible de différences, lalangue, comme "fonction d'excès » se dessine comme « l'ensemble de toutes les chaînes possibles, celles que la science représente : étymologie, paradigmes divers, dérivations, transformations, etc., et celles qu'elle récuse : homophonies, homosémies, palindromes, anagrammes, tropes et toutes les figures imaginables de l'association ».

$100 C f$. Milner (1978: 104) évoquant lalangue comme « une foule d'arborescences foisonnantes, où le sujet accroche son désir, n'importe quel nœud pouvant être élu pour qu'il fasse signe ».

101 Sur la fréquence de la métaphore musicale pour évoquer le discours de l'inconscient d'un sujet pensé comme " effet de langage », je renvoie à la présentation élémentaire de la parole hétérogène dans cette perspective psychanalytique in Authier-Revuz (1982b : 125-136) : au « tout discours s'avère s'aligner sur les plusieurs portées d'une partition », posée, en 1957, par Lacan dans « L'instance de la lettre dans l'inconscient » (repris in Lacan 1966 : 493-526), répond, par exemple, l'éclairant filage métaphorique par S. Leclaire du « chant de l'inconscient [...] » comme ne relevant pas du « contrechant d'une fugue ou [des] harmoniques d'une ligne mélodique » mais bien plutôt de la discordance d'une «musique de jazz que l'on entend malgré soi derrière le quatuor de Haydn écouté à un poste de radio mal réglé [...] » - faisant écho au dire dispersé en « voix multiples » évoqué ci-dessus 2.2, p. 516. 


\subsection{2 Échecs à la parole « de soi » : « paroles imposées » ou le langage qui «parle » tout seul}

Observer, dans la diversité des troubles du langage, les ratés, graves, d'une non-advenue d'une parole « de soi »- c'est-à-dire habitée comme sienne par un sujet parlant - atteste que, si commune que soit l'issue heureuse du processus d'appropriation, il y a - dans la contradiction inhérente à l'extériorité interne comme un « défi » à relever pour se «faire source » à partir de ce qui est reçu : les difficultés, les impossibilités que manifestent les troubles, font apparaître « en creux » ce que le sujet parlant " ordinaire » a dû impérativement réussir pour, dans l'aventure qui, par là, se révèle « à risque », prendre la parole.

Ainsi, dans le rapport altéré au langage qui est celui de la psychose ${ }^{102}$, des manifestations témoignent d'une pathologie langagière du rapport dedans/ dehors : lorsque de façon spectaculaire des voix s’imposent au sujet ou que, plus discrètement, la parole qu'il émet apparaît comme " plaquée » ou « citée ${ }^{103}$ : cela montre que le sentiment d'énoncer une parole de soi peut, pour le sujet, demeurer, dans la souffrance, hors d'atteinte ou très fragilisé, projetant un éclairage sur la parole « normale » quant à ce qui relève de son placement énonciatif dans le réel de l'extériorité langagière.

Sous des noms divers - celui de son baptême en " automatisme mental » par Clérambault ${ }^{104}$, ou ceux de " voix intérieures », « paroles imposées », " écho de la pensée », « hallucination verbale »... - le trouble qui consiste en ce que familièrement on appelle " avoir des voix " a fait l'objet, du $19^{\mathrm{e}}$ siècle à nos jours, de très nombreux travaux. Il n'est ni de ma compétence ni, j'espère, de ce que requiert mon objet, d'esquisser un panorama de cet ensemble, riche et complexe, d'investigations psychiatriques et psychanalytiques - observations cli-

102 Cf., par exemple, dans leur développement différencié : Lacan (1966 : 531-589), (1981), Manier (1995) et Soler (2008), (2015).

103 Étant entendu que les troubles langagiers psychotiques ne se limitent pas à ceux que nous évoquons ici en tant qu'ils semblent entrer en résonance avec la question - posée par la RDA et sa fonction dissimilatrice - de l'énonciation d'une parole de soi dans le réel de l'extériorité du langage : le rapport altéré au langage de la psychose peut aussi, notamment, se manifester dans la prolifération signifiante - équivoque, néologismes, mots-valises, glossolalies - d'une parole qui, livrée sans bornes au réel langagier de la substance signifiante, « dé-chaîne », selon le mot de L. Irigaray (1974) les mots de la langue, comme, différemment, J.-P. Brisset (Euvres complètes, 2001) ou le Finnegans wake de Joyce en donnent des exemples. Sur l'observation des « tonalités récitatives » chez « un sujet en phase schizophrénique », voir Schepens (2002b : 11-31).

104 Gaëtan Gatian de Clérambault (1872-1934), psychiatre, médecin-chef de l'infirmerie spéciale [...] de Paris, clinicien que J. Lacan reconnaissait, en 1966, comme son « seul maître en psychiatrie ». 
niques et réflexions théoriques -, tant dans sa dimension historique que dans la diversité ou l'opposition des approches du phénomène (touchant notamment à la causalité organique ou psychique de celui-ci, articulées à des conceptions pré- ou post- freudiennes, et repensées dans le cadre du « sujet effet de langage » de Lacan) $)^{105}$.

Je ne m’attacherai pas non plus à détailler la gamme étendue des formes prises par cette « xénopathie langagière »; je me contente de rappeler la diversité des manifestations de l'emprise d'une voix « étrangère » sur le sujet ${ }^{106}$ pour souligner que ce trouble ne se ramène pas au délire d'un sujet en proie aux paroles persécutrices ou injonctives de quelque voix de Dieu ou de diable ; ce sont aussi des voix neutres, affectivement, qui s'imposent au "récepteur ». Lorsqu'elles accompagnent la vie du sujet d'un incessant " commentaire de ses actes », par exemple, elles ne sont pas nécessairement plus critiques ou menaçantes que la très normale « petite voix » intérieure susceptible de s'exclamer en « je » ou en « $\mathrm{tu} »$ :

- Ah, ce que je suis bête, j’ai encore oublié de...

Ça y est, tu t’es encore trompée, le jour où tu seras capable de lire correctement un horaire...

Mais, voix « d'ailleurs » à l'intérieur du sujet, elle parle de « lui/soi » à la troisième personne, ou bien elle «le/se » désigne par « on » :

elle fait ci, elle fait ça ${ }^{107}$

on veut répondre, [...] on attendra bien un peu. ${ }^{108}$

105 On trouvera - et j'utiliserai ci-après - des éléments précieux sur l'histoire et l'actualité de cette pathologie dans des publications récentes : E. Porge (2012) qui en rappelle, en ouverture de sa réflexion aigüe sur la voix (p. 19-25 notamment), des étapes cruciales : G.-G. Clérambault, H. Ey, D. Lagache, J. Lacan... et le Journal Français de Psychiatrie qui consacre un numéro entier ( $\mathrm{n}^{\circ}$ 45, Faucher et al. (dir.) 2018) à un premier parcours - historique et clinique - de l'automatisme mental.

106 Entre autres : énoncés suivis ou « bribes », énoncés interrompus donnés à compléter au sujet ; voix entendues comme émanant de personnes présentes mais silencieuses, ou d'écrits affichés ; voix seulement entendues ou « émises » par le sujet...

107 C. Soler « Qu'est-ce que la psychose ?» France Culture, Chemins de la philosophie, 15-12-2016. 108 Noté par Clérambault (2 avril 1920) au sujet d'un malade : "Automatisme mental. Scission psychique. Voix intérieure qui l'inhibe et se substitue à sa pensée. [...]. Parle d'elle-même à la troisième personne : “on” [...] » (reproduit in « Bibliothèque Confluents », Association de la Cause Freudienne- Île de France, Printemps 2004). 


\section{(i) Paroles qui s'imposent, sans émetteur...}

Au niveau « basique » où je me situe, je voudrais simplement souligner les deux traits - d'ailleurs fortement solidaires - qui se trouvent unanimement relevés à propos de cette pathologie de l'extériorité langagière, quelles que soient la variété de ses manifestations et la diversité de ses approches : le caractère de ces voix pour le sujet : 1) imposé et 2) incertain quant à leur source...

On est frappé, en effet, de la récurrence du vocabulaire par lequel patients et soignants, de Clérambault à nos jours, disent la violence éprouvée par ceux qui se plaignent d'avoir « des voix dans la tête ». Reviennent en leitmotiv le sentiment pour le sujet d'être le siège d'un phénomène mécanique, comme celui d'un « écho », intérieur, d'un « automatisme » mental, d'être soumis, dans son intériorité, à une «force extérieure » :

une force qui le commande... forcés à penser par l'influence de forces extérieures... sujets qui se plaignent de ce qu'on les force à parler par pensée ou par action... paroles imposées... phrases qui s'imposent... sa pensée lui est imposée... prise de la pensée du malade par autrui...

ressentie comme une intrusion

intrusion de l'autre en moi... force envahissante... parasite... processus qui prennent possession de lui... assailli... harcelé... investi... ${ }^{109}$

Rejetant en dehors de lui cette intrusion langagière, le sujet n'en éprouve pas moins qu'il en est le siège, le lieu, le théâtre... Ce qu'il y a de contradictoire ou d'énigmatique au plan de la subjectivité dans le phénomène que Clérambault ${ }^{110}$ met au jour comme « automatisme mental » est d'emblée au cœur de la description qu'il en donne (ici, résumée par E. Porge (2012 : 22-25)) :

« on me donne des pensées qui ne sont pas à moi ». [...] Le sujet accepte qu'il s'agisse de sa pensée mais il la perçoit comme imposée du dehors. Il est dédoublé face à sa pensée.

(p. 23)

$\mathrm{Au}$ regard de ces formes verbales un dédoublement se produit pour le sujet ; à la fois il s'agit de sa pensée et elle lui vient $d u$ dehors, elle lui est imposée de façon parasitaire [...] (p. 24)

109 Fragments émanant des malades ou des cliniciens relevés au fil des pages dans Faucher et al. (2018).

110 G.-G. de Clérambault, Euvre psychiatrique, t. I et II, PUF, Paris, 1942. 
Contradiction que $\mathrm{H}$. Ey ${ }^{111}$ reformule en notant que la victime de " voix intérieures » « en pensée » a l'impression de « ne pas être l'auteur du discours pourtant intérieur qu'il entend ».

Aux antipodes du sentiment d'être source, auteur, de sa pensée et de son dire, l'expérience du sujet est celle d'une radicale - vertigineuse - incertitude énonciative $^{112}$ où vacille l'ancrage identitaire : le "ça parle/pense dans ma tête » débouche sur un "je ne sais pas qui pense/parle », moi ou un autre. De la dépossession subjective qu'entraîne pour le sujet l'origine incertaine - soi/pas soi ? - de ce qui pense/parle en lui, C. Soler (2008 : 127-133) présente l'exemple d'une jeune femme psychotique, « Marlène », dont « les voix » sont le symptôme majeur :

Lorsque la question «Qui le dit ? » lui est posée, elle répond d'un geste évasif. [...] elle a indiqué s'être posé elle-même la question [...] « Était-ce moi ou l'autre ? » La réponse reste cependant hypothétique, instable, quasi indifférente. (p. 129) ${ }^{113}$

Pour Marlène, les voix, plurielles, ne font pas un émetteur. Elles présentifient une sorte d'émission omniprésente, presque coextensive au bain signifiant. (p. 129)

Loin que, dans le vécu de cette troublante expérience langagière, une parole adressée puisse prendre corps, et avec elle, le sujet qui l'énonce -

Pour Marlène, au vide de l'énonciation du côté des voix, répond de son côté le défaut d'identité et l'indétermination de son être. (p. 130)

le langage est, pour le sujet, un réel qui le traverse :

Il y a $d u$ message mais pas de message. (idt) (p. 129) $)^{114}$

111 H. Ey, Traité des hallucinations, t. I, Masson, Paris, 1973. Cité d’après Porge (2012 : 20).

112 Incertitude pathologique qu'il faut distinguer des flottements ponctuels éprouvés normalement par les sujets, si agacés ou troublés qu'ils soient de rencontrer, au détour d'un mot, d'une expression, le « parlé » de leur propre parole ( $c f$. ci-dessus chap. 11 2.3.3, p. 455 et, ci-dessous, 2.3.4).

113 À ce flottement du sujet quant à ce dire « en lui », E. Porge (2012 : 36) fait écho lorsqu'il note « la difficulté à en parler » : « La façon dont quelque chose se dérobe toujours de façon singulière quant il s'agit pour le délirant de communiquer sur la nature de ses voix [...] qui se traduit parfois au seul fait que "ça” s'impose à lui ».

114 De la même façon, C. Fromentin, in Faucher et al. (2018 : 55), analysant ce qu'il en est pour un sujet de la voix chargée d'insultes qui « s'impose à lui » - « Le sujet ne s'y reconnaît pas. Il sait [...] qu'il en est le support, à la fois émetteur et récepteur, mais il ne peut s'attribuer cette insulte au sens où il en serait l'auteur » - conclut : « Il s'agit d'un énoncé coupé de son énonciation ». 
Remarque 7 : Subir des « paroles imposées » et représenter les « idées reçues ». " [...] en état d'estrangement devant les mots : c'est le dehors passé à l'intérieur, c'est l'intérieur saisi comme extérieur » : le diagnostic que Sartre (1988) porte, en frère de souffrance ${ }^{115}$, sur l'auteur de Bouvard et Pécuchet, pourrait concerner une victime des paroles imposées, marquant ce qu'il y a d'intime parenté entre cette pathologie et l'entreprise esthétique de Flaubert, visant un Livre « entièrement recopié », et le commentaire de Barthes (1970) au sujet de Flaubert

On ne sait jamais s'il est responsable de ce qu'il dit (s'il y a un sujet derrière son langage) ; car l'être de l'écriture (le sens du travail qui la constitue) est d'empêcher de jamais répondre à cette question : Qui parle?»

consonnent de façon troublante avec les analyses évoquées ci-dessus. Mais, si « insensée », « vertigineuse » qu'elle soit (selon toujours les mots de Barthes), l'écriture - ô combien différente de sa pratique épistolaire où s'affirme un énonciateur robuste - relève d'une ascèse du répété que pratique un créateur de textes qu'il signe. On revient (chap. 14) sur cette position énonciative extrême, risquant l'effacement de la parole propre du sujet, au profit d'une représentation - généralisée - du discours autre que porte et organise, invisible, une instance - écrivante - souveraine. Il importait ici, seulement, de marquer qu'il y a antinomie, précisément au plan énonciatif de la RDA, entre - si proches soient-elles, en même temps - les « paroles imposées » que subit un sujet et les « idées reçues » dont un autre compose un « Dictionnaire ».

\section{(ii) altération non de la langue mais de l'énonciation}

Les voix, que les sujets les « extériorisent » ou qu'ils « en parlent », ne maltraitent pas le système de la langue ${ }^{116}$ : ce sont des chaînes normalement constituées au plan linguistique qu'ils émettent. La maîtrise de la langue n'est pas touchée, mais « seulement » - si l'on peut dire - la capacité énonciative d'y inscrire subjectivement une pensée et une parole éprouvée comme « propre ».

J.-M. Faucher note ${ }^{117}$ que le père de l'automatisme mental, Clérambault,

estimait justifié de le remarquer : «La pensée qui devient étrangère le devient dans la forme ordinaire de la pensée».

De cette observation, commune, de la normalité linguistique des « voix », on peut rapprocher ce qu'on a évoqué plus haut comme manifestation plus « discrète »

115 G. Philippe (1997 : 188) formule ainsi le « véritable drame du langage chez Sartre [...] : nous ne pouvons nous exprimer qu'à travers les mots des autres », $c f$. Ndiaye (1992 : « Roquentin et la parole vierge »)

116 Mis à part les cas où, évoluant en délire, les voix reçues, attribuées à des êtres divins ou diaboliques... - comme, par exemple le « président Schreber » auquel Dieu s'adressait en « langue fondamentale ».

117 Dès l'éditorial de Faucher et al. (2017) 
- sans « voix » - du rapport altéré au langage de la psychose. Dans son approche clinique A. Manier (1995) observe, dans l'expression de patients psychotiques, la conjonction d'un fonctionnement grammatical correct, voire élégant ou même virtuose $^{118}$... et d'une émission verbale reçue par lui comme

simplement phonée - comme une citation - par le psychotique, simple relai et haut-parleur (p. 120) $)^{119}$

relevant

d'une espèce de "plaqué langagier », de simple vernis de représentation langagière présent chez à peu près tous les psychotiques (p. 109).

De ce qu'il caractérise comme une « apparence de fonctionnement du langage » (p. 88), A. Manier rend compte à travers l'opposition entre le « je parle » du sujet habitant le langage et le «ça phone » du psychotique que traverse le langage « tournant » en lui comme un moteur à vide, sans que s'y produise l'embrayage d'une énonciation.

Parler du « phoner » du psychotique ne signifie pas (« Un psychotique, pour être psychotique, n’est pas nécessairement seulement psychotique » souligne A. Manier (1981 : 82) que le régime d'expression d'un psychotique soit continuement celui de sa « phonie » : moments de phonie et moments de parole peuvent se succéder pour une même personne, la capacité du thérapeute à « distinguer ce qui est langage de ce qui est phonie » (p. 159) étant, justement, essentielle dans la perspective, qui est celle de l'auteur, d'une « linguothérapie » tentant de faire accéder le sujet à un « fonctionnement langagier » en s'appuyant à ce qui a pu - si limité soit-il - s'en établir, présent dans des « moments de langage fonctionnant selon le code social » (p. 82).

Remarque 8 : Un cas de parole « comme citée ». La problématique du caractère " comme cité » d'une parole psychotique apparaît au cœur de l'observation détaillée du discours d'un enfant par E. Filhol. Troué par instants de brèves irruptions de parole propre (« Moi je ne suis pas fou. J'ai eu des ennuis avec ma famille » (Filhol 1997 : 79), le discours qu'il « déroule » - dans

118 Par exemple p. 160, ou p. 168 : « Jamais psychotique ne me fit mieux comprendre la différence entre langage et phonie : son français était élégant et impeccable mais ne comportait pas la moindre trace de langage. [...] En lui la phonie phonait des idées comme un moteur tourne à vide ».

119 Cf. ci-dessus, Remarque 3, p. 507, les observations (faites dans le cadre de thérapies d'enfant) de paroles « échoïques ", " inexpressives », ou encore comme en " marionnettes de l'Autre » (Soler, 2008 : 80). 
un tragique régime d'aliénation - est clairement identifiable comme celui d'un père follement « contrôleur ${ }^{120}$, dont il n'est que le relais sonore. Cette parole dépossédée d'elle-même - à laquelle le « changement de voix », d'un débit plus rapide, précipité, « appose [...] le sceau d'une étrangeté » - n'est pas une parole qui cite, c'est-à-dire qui ferait apparaître en elle, disjointe, une parole autre, dont elle parlerait.

C'est pourtant comme " citation ${ }^{121}$ que - en toute rigueur de façon impropre - sont évoqués dans l'étude les énoncés

qui ne cessent pas d'envahir le discours, obligeant celui qui les énonce à les proférer comme si c'étaient ses propres paroles (ibid. : 81);

le décalage du « comme cité » - qu'on dirait cité mais qui ne l'est pas - prenant, dans cette analyse de « la citation du psychotique » (ibid. : 86), la forme paradoxale d'un recours régulier au terme de " citation », assorti de commentaires déniant ce statut ${ }^{122}$ au référent ainsi nommé :

des citations empruntées au discours de l'autre mais qui n’apparaissent jamais en tant que telles par celui qui les cite (ibid. : 81)

un autre texte cité, non désigné comme tel, à l'insu du sujet qu'il parle et qui le parle (ibid. : 81)

Un élément vient cependant comme conforter l'usage du terme « citation » - si problématique qu'il apparaisse avec ce qu'il implique de distanciation prise dans la parole « se faisant » par rapport à une parole autre - c'est l'apparition énigmatique, répétée en point d'orgue de coulées de discours paternel passant sans distance dans la parole de l'enfant, de la formule : « Fin de citation".

Dans son étrangeté, le fait que rétroactivement elle caractérise comme « citation » le flux langagier qui précède peut interroger le caractère radical de la ventrilocation dont le sujet est victime, y faisant surgir l'ombre d'un dédoublement méta-énonciatif de RDA, susceptible d'être perçue comme fragile et incertaine tentative d'accès à une parole de soi ${ }^{123}$.

$120 \mathrm{Cf}$. «Moi, vous savez, dit le père, [...] rien ne m’échappe, je sais tout ce que peut faire Frédéric. On ne peut rien me cacher, je suis comme le serpent qui s'insinue partout [...] Qu'est ce qu'il vous a dit? Moi je vais le faire parler.» (p. 81)

121 «Le discours de Frédéric, à certains endroits, n'est pas son propre discours mais une citation de la parole paternelle »; « aliénation à la citation de l'autre » (p. 81, 82)

$122 \mathrm{Si}$ on prend " citation " au sens strict ( $c f$. chap. 9 Appendice : Citation I p. 361) de reprise intentionnelle marquée (ou donnée à reconnaître) comme telle. En revanche, dans son extension au réel du déjà-dit dans le dire, le sens IV de citation (comme son dérivé « citationnel ») pourrait rencontrer cette parole comme relevant d'une « citationnalité pathologique ».

123 Interprétation sur laquelle - avec une prudence modalisante en peut-être et conditionnel hypothétique - s'achève l'étude de Filhol (1997), envisageant que cette « fin de citation » « pourrait signifier [...] l'émergence d'une parole personnelle, nouvelle, moins aliénée ». 
Exemplairement dans ce qu'on appelle « paroles imposées », comme, plus discrètement, dans ce qui s'articule « comme cité », il y a absence de parole - au sens de l'investissement énonciatif d'un sujet ${ }^{124}$; théâtre de voix étrangères, support de " plaqué langagier », le sujet n’a pas partie liée avec le langage qui lui demeure extérieur, fait - et corps - étranger, non intériorisé, assimilé, qu’il laisse « passer par lui » sans y « mettre du sien ».

De cette pathologie langagière qui, inscrite dans le fonctionnement de l'extériorité interne du langage, empêche qu'advienne une parole - un discours, un dire - on peut rapprocher deux formulations : celle d’A. Manier (1995 : 59) qui la saisit comme « langue privée... de langage » et celle de C. Soler évoquant ${ }^{125}$

«le hors-discours de la psychose» (2008:65),

«le schizophrène hors discours - mais pas hors langue » (2009: 195),

« discours » désignant ici « une modalité du lien social en tant qu'ordonné par le langage ${ }^{126}$, « lien », « ordonnancement » qu'assure l'usage socialement partagé du langage, sans lequel le psychotique est « lâché » dans un monde aux limites et séparations non-établies.

Remarque 9 : Parole pensée vs phrases émergentes : un témoignage aigu de vacillement identitaire. Dans le long entretien entre J. Lacan et un patient souffrant de " paroles imposées ${ }^{127}$, la frappante acuité avec laquelle celui-ci parle avec ses mots à lui de l'expérience angoissante d'être le lieu où se produit - «émerge » dit-il - du verbal ${ }^{128}$, en fait un document remarquable. Le phénomène est répétitivement saisi par lui comme

ce qui (« ça, cela ») vient, émerge, s’impose.

Apparaît une « répartition » marquée, au plan grammatical, de la première personne : massivement présente (pronom ou déterminant possessif) en fonction de complément (direct, indirect, de lieu...) comme dans

124 Et au sens où J. Lacan (1966 : 279-280) reconnaît « une absence de la parole » « dans la folie » « où le sujet, peut-on dire, est parlé plutôt qu'il ne parle. »

125 Dans le sillage de Lacan (1977) « L'étourdit».

126 Soler (2008: 63) ; ce qu'on peut rapprocher du retour de A. Manier (1991) sur le caractère crucial de l'accès - ou non - au-delà de la langue, au langage socialement codé (p. 57), c'est-à-dire à la dimension sociale - partagée, impliquant contrat, confiance... - de «l'usage » du « fonctionnement, de la « fonction » du langage (p. 49, 53, 115...).

127 Entretien du 13-02-1976 dont le verbatim est publié dans Faucher et al. (2018b) (et je remercie ses coordinateurs de me l'avoir communiqué). Lacan revient sur cet entretien ( $c f$. ci-dessous 2.3.3, p. 531) dans le séminaire qui suit (année 1975-76), cf. Lacan (2005 : 95).

$128 C f$. «Cela vient d'un seul coup : “vous avez tué l'oiseau bleu”, “c'est un anarchic système”..., des phrases qui n’ont aucune signification rationnelle dans le langage banal [...]. » 
ça me vient ; elles m'envahissent

s'impose à moi/à mon cerveau/à mon intellect

elle n'apparaît en je que 3 fois dans la construction étrange (comme on dit « j’ai une poussée d'acné, des élancements... ») :

j’ai des phrases émergentes/imposées ; j’ai une phrase imposée qui dit

ou en contexte de procès passif ou négatif :

je suis un peu disjoint du point de vue langage ; comme si j'étais peut-être manipulé... je n’arrive pas à expliquer ; je ne sais pas comme elle vient [...] cette émergence

La place des actants sujet des procès s’imposer, émerger, venir... étant occupée par phrase(s) ou émergence, incessamment repris anaphoriquement par elle(s), ça, cela

Des phrases qui s'imposent ; ce sont des phrases qui émergent ; l'émergence s'impose à moi ; ces phrases imposées ; dans la mesure où elles émergent pour...

De façon remarquable, le terme parole (imposée) n’apparaît pas chez le patient, sinon, plusieurs fois, en reprise immédiate d'un propos de Lacan

Dr Lacan : [...] Les paroles imposées sont d'avant ? / G.L. : Les paroles imposées [...] ont commencé en mars 1974

C'est au terme de «phrase » qu'il recourt pour désigner « ce qui émerge » au plan langagier, usage relevant d'un choix affirmé par rapport à « parole » qui se manifeste dans la succession reprise interlocutive/reformulation

Dr Lacan : [...] Qu'est-ce que vous appelez la parole, que vous dites, vous, la parole imposée?

G.L. La parole imposée, c'est une émergence qui s'impose à mon intellect et qui n’a aucune signification au sens courant. Ce sont des phrases qui émergent [...] qui ne sont pas déjà pensées [...].

ou, plus nettement encore, en rectification catégorique

Dr Lacan : Alors, c'est vous même qui le dites, ces paroles...

G.L. : Non, ce sont des phrases.

G.L. revient, à plusieurs reprises, sur la différence qu'il éprouve entre les deux régimes langagiers de sa parole et de l' « émergence » de phrases dont il est le siège, qu'il caractérise, négativement, par rapport à la première, comme « non-réflexives », « pas déjà pensées », leur succession sur la chaîne lui apparaissant comme

une disjonction entre une phrase imposée et une phrase de moi. 
Et c'est par ce terme de disjonction passant à travers lui qu'il évoque cette angoissante cohabitation :

Je suis un peu disjoint au point de vue langage, disjoint au niveau du langage. [...] Il se fait une disjonction.

débouchant, au plan identitaire dans le trouble, l'incertitude, sur la plainte, énoncée d'entrée de jeu (en réponse à un « parlez-moi de vous ») :

Je n'arrive pas à me cerner.

reprise en clôture d'entretien, en forme d'appel au secours :

J'ai un tel espoir de retrouver mon pouvoir de jugement, mon pouvoir de dialogue, un pouvoir de prise en main de la personnalité. Je crois que c'est le problème crucial. Comme je vous l'ai dit au début, c'est que je n'arrive pas à me cerner, je n'arrive pas à me prendre en main.

\subsubsection{Comment peut-il se faire que... la parole advienne/n'advienne pas ?}

Deux réactions de questionnement étonné devant une (non)advenue de la parole éprouvée comme propre, deux « Comment peut-il se faire que... ? » se font face :

- celle de D. Lagache, à l'orée de son ouvrage sur les hallucinations verbales

Comment la parole propre peut-elle apparaître à son auteur comme une parole étrangère ? ${ }^{129}$

pointant le caractère communément perçu comme insensé, aberrant, de la dépossession ventriloque ressentie par le sujet, méconnaissant ce qu'il produit,

- et celle, paradoxale et souvent citée, de Lacan ${ }^{130}$, s'interrogeant à l'inverse sur le mystère qui nous fait- normalement - méconnaître le réel que, dans sa « rigueur ${ }^{131}$, rencontre le psychotique :

Comment est-ce que nous ne sentons pas tous que des paroles dont nous dépendons nous sont, en quelque sorte, imposées ? C'est bien en quoi ce qu'on appelle un malade va quelquefois plus loin que ce qu'on appelle un homme bien portant. La question est plutôt de

129 Page 1 de Les Hallucinations verbale et la parole, Librairie Felix Alcan, Paris, 1934, cité in Porge (2012: 19).

130 Lacan (2005: 95), $c f$. note 127 ci-dessus.

131 « La psychose est un essai de rigueur » dit Lacan (Scilicet 6/7, 1976, p. 9), cité in Roudinesco (1993 : 485). 
savoir pourquoi un homme normal, dit normal, ne s'aperçoit pas que la parole est un parasite, que la parole est un placage, que la parole est la forme de cancer dont l'être humain est affligé.

C'est cette interrogation - provocativement négative - que reprend C. Soler (2008), en la reformulant sur son versant positif, lorsqu'elle se demande :

comment ce sujet défini comme pur effet parlé par l'autre, peut virer à l'agent, devenir quelqu'un qui parle, qui désire, autrement dit quelqu'un qui s'anime de libido (p. 67)

et, au delà

ce qui protège le névrosé de l'expérience énigmatique que le psychotique rencontre (p. 121).

Les troubles ou échecs de la parole propre questionnent et éclairent, en effet, quant à ce que celle-ci requiert derrière l'évidence de son fonctionnement ordinaire. Il importe, pour en approcher quelque chose, de bien distinguer les deux plans où peut s'énoncer et signifier un «ça parle » :

a) celui de l'élaboration théorique, pertinente, du fait de l'extériorité interne du langage - qu'elle se formule dans le principe du « ça parle toujours, ailleurs, avant et indépendamment » ou dans les diverses réflexions évoquées ci-dessus (2.2) - qui, posant conceptuellement un réel sous-jacent au « je parle », questionnent la souveraineté énonciative et la prétention de ce « je » à être source et origine,

et b) celui du vécu, pathologique et souffrant d'un « ça parle dans ma tête ", antinomique du sentiment d'énoncer - en « je parle » - une parole propre.

À s'éprouver - au risque que s'y perde, dans le brouillage des frontières dedans/dehors, le vital sentiment des limites de soi et de son corps - comme traversé (envahi, investi, assiégé...) sans défenses par le flux du langage, le sujet psychotique, témoin rigoureux- martyr ( $c f$. note 69 p. 509) - de l'extériorité d'un langage qui nous est imposé, y perd le pouvoir de faire sienne une parole énoncée comme propre.

Prendre la parole suppose en effet d'être - paradoxalement - en mesure « d'aller moins loin » que le psychotique dans sa vertigineuse et invivable «lucidité ", d'être à même de "ne pas s'apercevoir » du «ça parle » qui nous traverse, c'est-à-dire de lui opposer la force positive d'une méconnaissance. C'est celle-ci, salutairement protectrice, qui fait défaut au psychotique, le livrant désarmé au flux du langage ; cette méconnaissance n'est pas de distraction ou d'inattention... mais ce que le sujet parvient à produire, à initier et à maintenir : la vitale consistance imaginaire d'une parole qu'il énonce, comme 
sienne ${ }^{132}$, c'est-à-dire qu'il pose (impose) comme « faisant corps » dans (contre) le réel dispersant du flux langagier qui le traverse.

Le miracle ordinaire de la parole est celui de la conversion qu'elle opère du règne de l'extérieur d'un « ça parle » dans l'engagement énonciatif du sujet dans le « dedans » d'une parole de soi. Si elle requiert l'élan d'un désir de parole, une confiance accordée au langage, cette conversion, qui permet de " faire sien » le langage, fonctionne " à la méconnaissance », s’alimentant à la puissance de l'imaginaire. Avec M. Schneider (1985 : 241), rappelons que, si le « propre » de la pensée, de la parole, est « sans doute à situer dans le registre des illusions », il vaut, comme tel, «ce que valent les illusions : beaucoup. »

On verra, au chapitre suivant, comment on peut considérer la RDA - partie prenante de l'opération de conversion du «ça parle » en un « je parle parmi d'autres » - comme rouage du mécanisme de méconnaissance protégeant la parole.

Dans leur diversité, les faits rassemblés ci-dessous, évoqués de façon sommaire, le sont en tant que, à leur façon, ils conjoignent tous, ponctuellement, RDA et «question » - trouble, manque, conquête, défense - d'une parole de soi, témoignant de leur articulation dans l'économie énonciative.

\subsection{4 « Histoires » de RDA et de parole propre}

On a aperçu ${ }^{133}$ comment, dans le processus normal d'acquisition ou dans le déroulement chaotique d'une parole dépossédée d'elle-même, la moindre émergence - même incertaine - d'une distance prise à « de l'autre » dans sa parole était saluée - ou espérée - comme un pas vers l'advenue d'une parole propre. Mais nombreux et divers sont les « faits » de RDA qui, au fil d'un dire adulte et normal - ayant toujours affaire constitutivement à l'altérité langagière dont il est tissé -, témoignent du besoin du sujet énonciateur d'assurer, en y repérant « de l'autre », sa parole propre : ainsi de ces « histoires de RDA » que recèlent aussi bien la parole la plus ordinaire (i) que ces deux pratiques intenses du langage, la parole en psychanalyse (ii) et l'écriture littéraire (iii) qui, selon M. Schneider « dénoncent mais sans cesse réénoncent la revendication du propre » (1985: 256).

$132 C f$. P. Arel, in Faucher et al. (2018a : 63) évoquant comment «l'homme parasité par le langage qui le conditionne dès les premiers jours [...] parvient à se constituer une petite place qui lui laisse l'illusion qu'il a pris la main, en usant de la parole. »

133 Cf. ci-dessus Remarque 3, et 2.3.2 (ii) Remarque 8 « Fin de citation », p. 528. 


\section{(i) Petites aventures de l'ordinaire du dire}

Communes au fil du dire, évoquées plus haut, chap. 11 (38-40), elles se jouent en deux temps : la surprise de l'énonciateur -Tiens... ; Voilà que... ; Merde alors..., etc. - à éprouver que, soudain, sa parole lui échappe au profit d'un autre, à laquelle, aussitôt, répond une RDA qui, identifiant et jugeant la voix clandestine, reprend, en surplomb méta-énonciatif, le contrôle sur un dire brièvement perturbé.

À noter que le retour à la parole éprouvée comme propre ne passe pas exclusivement par le rejet de mots importuns dont une RDA stigmatisante « débarrasse » le dire, mais aussi (moins souvent) comme dans :

- Viens voir, il y a au fond du jardin une touffe de dahlias de toute beauté... Tiens, je dis comme Maman... tu te souviens?

- Mais elle disait autrement : « de-tou-te-beau-té »...

- Ben, dis, ce n'était pas de l'imitation ! [rires] (oral, entre deux sœurs, 15-08-15)

où la RDA répondant à la surprise de reconnaître inopinément dans sa parole un déjà-dit non-convoqué, débouche plutôt dans un accueil attendri de cette voix autre, comme un harmonique bienvenu aux mots de l'énonciateur (proche, sur un mode implicite, du partage complice d'un comme disait Maman).

Peu importe, dans ces menus évènements (méta)-énonciatifs, la couleur - hostile ou accueillante - de la réponse apportée par la RDA au trouble d'une parole à se découvrir, ponctuellement, altérée, désunie par une voix étrangère : la fonction dissimilatrice de la RDA sépare explicitement l'autre de l'un, libérant de son emprise insue le dire qui se reprend, comme parole de soi.

\section{(ii) « Moments » de talking-cure}

Pourquoi vient-on chez un analyste [...] Sinon pour y dire cette seule plainte : je n'ai pas de mots qui me soient propres.

Sur ce constat de M. Schneider (1985 : 256) selon lequel, derrière le mal-être qui conduit à entreprendre une psychanalyse, joue le défaut d'une parole de soi, de nombreux psychanalystes, dans des cadres théoriques différents, se rejoignent. Dans cet accord, on retiendra les points suivants, largement partagés :

- le lien entre quête de « mots à soi » et quête identitaire « de soi ». Ainsi J.-B. Pontalis fait-il se répondre les deux versants de :

« Parler avec des mots à moi » disent-ils un jour ou l'autre, fatigués de tant de paroles ingérées, empruntées, incrustées dans leur chair. (1986: 98) 
et

[...] ce souci taraudant que nous avons de notre identité... [souffrant] qu'elle soit si incertaine, si précaire, si largement dépendante (ibid. : 175)

que M. Schneider conjoint dans ses « êtres palimpsestes » :

Les êtres ne sont-ils pas des palimpsestes, traces de mots effacés - jadis prononcés -, autres par les parents, les frères, médits, maudits, parfois surchargés d'altérations, livres effaçant sans cesse les arrière-textes dont ils sont faits (1985 : 310).

- la nécessité pour le sujet de son désir de mots à soi, pourtant inaccessible, vouant à un " autant que faire se peut ${ }^{134}$ ", aussi bien la quête, selon D.-R. Dufour (2016), de « ceux qui vont chez les psychanalystes pour essayer de parler en leur nom » (p. 126), que ce que - face à la « situation de radicale extériorité de l'humain au langage - O. Grignon (2017) estime être « le travail d'une analyse » : « rendre un peu plus viable » le rapport de quelqu'un au langage ${ }^{135}$.

Revendication absurde, certes, que d' « avoir » des mots à soi, rappelle Pontalis, mais

Pourtant, il faut qu'il mène à son heure ce vœu impossible [...]. Quand les mots manquent, c'est qu'à son insu, on s'apprête à toucher un autre sol. (1986 : 98)

Et si évidemment « chercher ses mots », et faire l'expérience de reconnaitre dans sa parole des déjà-dits qui, insus, y règnent comme chez eux, pour y découvrir le « sol » nouveau de mots à énoncer pleinement comme siens, n'est pas, heureusement, le privilège exclusif de la pratique psychanalytique ${ }^{136}$, celle-ci, par son dispositif spécifique de parole ${ }^{137}$, soutenu, dit A. Dufourmantelle, par

$134 C f$. aussi M. Schneider (2010 : 115) évoquant « l'idéal d'authenticité dont on s'approche en défaisant les paroles "comme si" [...] et en devenant, autant que faire se peut, un auteur plus qu'un porte-parole».

135 C’est-à-dire, ajoute-t-il (p. 92), « autant que faire se peut d'aller au plus loin de cet exil, si possible».

136 Comme en témoignent tous les « bougés » de parole qui se produisent, conscients ou non, dans le cours ordinaire de la vie d'un sujet, en rencontres et surprises, dans les flottements introspectifs, les rêves, la lecture des poètes, et tout simplement « l'usage de la parole » comme le rappelle N. Sarraute.

137 Celui d'une parole libérée des contraintes - et privée des protections - de l'usage communicationnel normal et soutenue par l'écoute d'un autre attaché à « tendre l'oreille au non-dit qui gîte dans les trous du discours » (Lacan, 1956 : 307). 
cette conviction héroïque que les mots nous sauvent si nous les faisons nôtres » («Au risque de la parole », $2011:$ 136),

vivifie la parole, catalysant sa capacité d'auto-écoute libératrice ${ }^{138}$.

Sur le chemin de mots « vers plus de mots de soi » qu'est une talking-cure, on peut noter que des « tournants » se produisent, qui passent spécifiquement par un fait de RDA. À côté des événements de parole, tels lapsus, homonymie,... jeux de lalangue qui, survenant au fil du dire, y font éclore un sens imprévu, des occurrences de RDA apparaissent, libératoires, comme moments clefs parfois dans l'avancée du dire d'un sujet vers $s a$ parole.

Remarque 10 : Récits de cure et RDA. Dans leur diversité ${ }^{139}$ les textes visant à rendre compte de la pratique psychanalytique présentent, sans surprise ayant un référent langagier, une forte densité de formes de RDA renvoyant aux dires des deux protagonistes.

Relevant d'un vaste espace générique ( $c f$. chap. 15. 1.1.1, p. 583) de discours « tenant lieu », ou « rendant compte » d'un autre discours, la réalisation spécifique qu'ils en offrent au plan des formes de RDA méritent une étude systématique (dont Sitri (2012) propose un élément).

Dans l'ensemble de ces " discours de RDA », le récit de cure affiche la particularité d'un fonctionnement « à deux étages » de la RDA : dans les propos représentés eux-mêmes s'observe la fréquence des faits de RDA, où se déploie la plus grande variété tant au plan des formes mises en œuvre qu'à celui des autres convoqués (je, vous, ma mère..., le journal, on, un poème...) que du statut référentiel des dires représentés (qui ont été dits, y compris en rêve, qui n'ont pas été dits, etc. parcourant toute la gamme évoquée au chap. 2.2.1, p. 39).

Ce n'est pas l'ensemble du riche fonctionnement de RDA dans le récit de cure qui nous retient ici, mais la part, très spécifique, des énoncés constituant, dans les propos des protagonistes, des « moments » d'avancée de leur parole, en quête « de mots propres », par la mise au jour, en elle, de discours autres clandestins.

« Histoires de RDA et de parole propre », ces énoncés le sont en ce que, ramenant à la surface d'une parole qui les y explicite, depuis les profondeurs où ils demeurent, insus et agissants, des discours fantômes - dits et oubliés, non dits et manquants - les RDA, en leur donnant corps, délivrent le sujet et sa parole de leur emprise cachée. En écho à la formulation de $\operatorname{Lacan}^{140}$ :

138 Que vivifie aussi la poésie, comme en témoigne Joë Bousquet remerciant P. Éluard : « Vous avez permis que je devienne celui qui dans ma voix m'écoute » (in P. Éluard Lettres à Joë Bousquet, 1973, p. 104, cité in Gantheret, 1996 : 173).

139 Écrits par l'un ou l'autre des acteurs du dispositif, avec des visées théoriques, cliniques, auto-biographiques, littéraires...

140 Dans « Fonction et champ de la parole et du langage en psychanalyse ». 
L'inconscient est ce chapitre de mon histoire qui est marqué par un blanc ou occupé par un mensonge : c'est le chapitre censuré. Mais la vérité peut être retrouvée ; le plus souvent déjà, elle est écrite ailleurs. À savoir : - dans les monuments [...] ; - dans les documents d'archives aussi : et ce sont les souvenirs de mon enfance, impénétrables [...] quand je n'en connais pas la provenance ; - dans l'évolution sémantique : [...] aux acceptions de vocabulaire qui m'est particulier [...] ; - dans les traditions [...] ; - dans les traces, enfin, [...] (1966: 259),

on peut dire que les énoncés de RDA évoqués ici restituent, en les représentant, de éléments de vérité retrouvée du chapitre censuré.

C'est ainsi le cas, classiquement observé, de paroles oubliées, très anciennes - interdictrices, jugeantes, assignatrices à une place ou un comportement, reçues parfois sans les comprendre, et dans la peur - enfouies, conservées dans cette forme vivante de la mémoire qu'est l'oubli, et dont le (parfois malfaisant) pouvoir souterrain s'évapore à l'air libre d'une parole qui les convoque, comme référents identifiés, localisés, maîtrisés par la représentation qu'elle en donne. On parlerait ici volontiers, pour ces RDA, de discours « rapportés », ramenés-remontés qu'ils sont du fond des âges et de la psyché où ils étaient enkystés, et remis en circulation de sens, dans le contexte d'accueil, neuf, d'une parole adulte, à même de se confronter à eux - devenus parfois dérisoires, de paralysants qu'ils étaient.

Je ne tenterai pas d'en évoquer rapidement des exemples, tant le récit de ces « remontées » libératrices ne prend de sens qu'à en suivre dans le plus grand détail l'imprévisible cheminement : c'est ainsi plus d'une dizaine de pages, (147159) auxquelles je renvoie, que F. Gantheret (1996), au chapitre Pouvoirs de la parole. L'interprétation, consacre à « seulement » raconter les étapes de la résurgence d'une parole (si profondément enfouie que le sujet ne sait même plus si «c'est un vrai souvenir » (p. 152) - résurgence qui achève de s'accomplir par une $\mathrm{RDA}$ ramenant la parole perturbatrice :

Ça m’a rappelé une parole de mon père à ma mère, à mon propos : « Mais non ! Elle n’a rien $\mathrm{vu} »$,

dans le hic et nunc de l'échange analytique où l'énoncé et la scène où il s'enchâssait sont désinvestis de leur pouvoir maléfique.

Autres discours fantômes, à même d'interférer gravement dans la vie de sujets, et dont ils peuvent, parfois, « se défaire » par la grâce de la RDA qui leur « donne corps » dans la parole qu'ils énoncent : des discours manquants - ceux qui, souvent dans des circonstances dramatiques de l'enfance ${ }^{141}$ ont été tus comme

141 Mort, suicide d'un proche masqué en « voyage » ou, à l'inverse, départ d'un père, d'une mère travesti en décès, filiation illégitime dissimulée, adoption passée sous silence, etc. 
autant de « secrets de famille ${ }^{142}$. Des mots ont fait défaut à l'enfant, laissant le sujet, " en souffrance » de ceux qui auraient pu/dû assurer la cohérence de son monde, dans le mal-être d'une discordance - un porte-à-faux entre son vécu et le discours environnant.

Un chemin de parole ${ }^{143}$ peut se faire, dans la cure, à travers les couches de discours venus en lieu et place des mots manquants au fil de ce que le sujet rencontre dans la sédimentation de sa mémoire, de trous, failles, flottements, impasses, contradictions..., comme autant d'indices dans un jeu de piste ${ }^{144}$ : son " débouché », via la RDA, dans le dire hic et nunc, de cela qui n’a pas été dit ${ }^{145}$, retournant par là vers le lieu ancien qu'occupaient mensongèrement d'autres discours, $\mathrm{y}$ " rapporte » la lumière des mots, pleinement énoncés, d'une « vérité retrouvée » et constitue un moment d'avancée de la parole propre hors de l'emprise de ces autres discours.

Ainsi, à représenter, au terme d'un parcours, un discours autre manquant, effacé ou jamais dit, c'est-à-dire en leur donnant corps (de mots), le sujet peut-il être à même, à la fois, de faire s'évanouir le discours « fantôme » et « la chose muette $^{146}$ » qui étaient en lui, et de prendre ses distances d'avec les discours autres qui en occupaient la place.

Notons que le mouvement qui consiste, pour un sujet, à faire taire ce qui, silencieusement, s'imposait dans sa parole et sa vie en lui donnant la parole - par le moyen d'une RDA qui, l'identifiant comme discours distinct de celui du sujet, en détache celui-ci - opère autant, pour dégager la voie à de la parole propre, au plan singulier de la vie affective (comme dans les cas évoqués ci-dessus) qu'à celui, collectif, social, des emprises idéologiques.

À l'opposé des discours manquants dont une RDA peut marquer la (ré) advenue, ce sont des discours conservés - et même comme immobilisés - en

142 Cf. ce rappel clinique de Lacan (1966 : 277) : « Nous savons en effet quel ravage déjà allant jusqu'à la dissociation de la personnalité du sujet peut exercer une filiation falsifiée, quand la contrainte de l'entourage s'emploie à en soutenir le mensonge ».

143 Dont le cours capricieux et complexe, emprunté singulièrement dans chaque cas, est aussi peu « racontable » brièvement que celui du chemin précédent ramenant à des mots oubliés.

$144 C f$. à la fin du passage cité p. 537, un des emplacements évoqués par Lacan où peut être retrouvée, « écrite ailleurs, la vérité du chapitre censuré » : « dans les traces, enfin, qu'en conservent inévitablement les distorsions nécessitées par le raccord du chapitre adultéré dans les chapitres qui l'encadrent, et dont [l'] exégèse rétablira le sens. » (1966: 259)

145 «P», mais c'est ce qu'on ne m'a jamais dit... ; Si on avait été capable de me dire «P»...; Il aurait fallu me dire que $P$, au lieu de...

146 « Chose muette », réduite à la « parole » somatique, et dont le sujet aspire, sur le mode du « Donnez-moi vos mots pour dire la chose muette qui est en moi » que M. Schneider (1985 : 324) prête à l'analysant, à se délivrer. 
mémoire, qui souvent, voire répétitivement, convoqués en RDA par un patient, vont soudain, à la faveur d'une nouvelle reprise dans un autre moment de la cure - et donc dans un contexte d'accueil modifié - se renouveler, en libérant un sens inaperçu.

Tel est le cas, par exemple, d'un parcours sur lequel F. Gantheret (1996) revient de façon précise et éclairante, où c'est une $R D A$ - la reprise par le sujet en modalisation autonymique d'emprunt, avec ses je-ici-maintenant, de mots biens connus des deux interlocuteurs - qui fait rupture libératoire, pour le sujet, dans la chaîne répétitive, où il se trouvait " pris », de transmissions littérales au DD, de cette parole figée, en « monument » dirait Voloshinov.

Ainsi ( $c f$. Remarque ci-dessous) la « redoutable efficacité » d'une « parole fatidique » qui « immobilise le sujet autour d'elle » - en l'occurrence autour de vœu de mort implicite dont elle s'avère, sous une forme dénégative, porteuse - se dissipe-t-elle dans le jeu énonciatif complexe d'une MAE : la réappropriation comme « siens » (en usage) des mots de l'autre, passant par la distance prise (en mention) avec ceux-ci, le sujet peut reprendre «à neuf » cette parole qu'il réanime et réouvre à la circulation du sens ${ }^{147}$.

Remarque 11 : « Historique » d'un parcours de RDA. Le chemin de parole évoqué ci-dessus passe par les étapes suivantes :

1. Paroles originelles adressée, au lendemain de l'hospitalisation d'urgence d'Émilie - future analysante, alors petite fille de quatre ans - par les médecins à sa mère :

Madame, votre fille est maintenant hors de danger, mais si vous n'étiez pas venue hier, vous n'auriez aujourd'hui que vos yeux pour pleurer.

2. Émilie entend d'innombrables fois, dans son enfance, le récit complaisant par sa mère de cet épisode s'achevant par cette phrase (vous n'auriez aujourd'hui...) que celle-ci se plaît à répéter.

3. Émilie évoque souvent dans l'analyse le souvenir de cette phrase et du malaise qu'elle éprouvait à l'entendre, sans en comprendre plus qu'autrefois la raison.

4. Émilie, de plus en plus souvent absente à ses séances, et en larmes, dit :

Je ne vous ai pas dit, mais je ne sais pourquoi mais j’ai failli ne pas venir hier.

5. L'analyste est saisi de l'écho de $1+2+3$, et je lui laisse la parole :

Je pensai, sans pouvoir me donner de raison ni même prendre le temps d'en chercher que je devais lui dire. «Si vous n'étiez pas venue hier », commençais-je. Elle acheva ma phrase,

147 Si la forme de la MAE se prête particulièrement bien à cette reprise d'un DD figé, il faut noter que c'est aussi en DD que, via le changement de contexte d'accueil, peut se produire la réactivation, hic et nunc, de l'énoncé « figé ». 
sans solution de continuité, sur un ton d'évidence paisible ; « ... je n’aurais aujourd'hui que mes yeux pour pleurer », dit-elle.

6. Cette séance, achevée par Émilie, souriante, « les yeux gais et secs », débouche, la nuit suivante dans un rêve « de mort », effrayant, et source d'apaisement en ce que 1) elle y échappe à la mort et 2) « ce n'était qu'un rêve ».

7. Empruntant avec humour le ton du conte pour achever son récit :

Émilie ne manqua plus de séance et ne fut plus jamais triste,

l'auteur note que - le questionnant, lui, sur le « comment » de ce miracle accompli par 4-5-6 - «le sombre mystère qui l'habitait l'avait quittée ».

\subsubsection{Pratiques de RDA au service d'une écriture propre}

Il ne s'agit pas ici de la variété des mises en œuvre stylistiques des ressources de la RDA - La Fontaine, Zola, Cohen...- mais de la réponse explicite apportée par certains scripteurs à leur souci - parfois leur hantise - de la prégnance du déjà-dit dans une écriture qu'ils veulent «propre » : celle du recours réfléchi à des outils de RDA, mis au service - défense ou conquête - de la singularité de leur écriture.

a) Du côté des stratégies de protection de son écriture - ses mots, sa pensée, son style... - la figure de Barthes s'impose, comme exemple d'une pratique, aussi constante qu'elle est revendiquée, de résistance, dans l'écriture, à la menace de la " grégarité » du langage, de l' « arrogance » de la Doxa, « régnant » sous les habits $\mathrm{du}$ « naturel $»^{148}$. Une vigilance méta-énonciative anti-doxa s'exerce au fil du dire, repérant les menaces d'intrusion de cette force invasive pour les « déjouer " par une prise, affichée, de distance.

C'est à la panoplie des outils à même de troubler la « bonne conscience » du dire d'un mot par le rappel du déjà-dit dont il est porteur que recourt l'« hygiène énonciative » ainsi mise en œuvre : univoques (ce qu'on appelle $X, X$ comme on dit, le $N$ appelé $X, \ldots$ ) ou interprétatifs (guillemets, italiques... ${ }^{149}$ ), formes de modalisation autonymique « d'emprunt » - qu'on pourrait dire ici d'emprunt « forcé » puisque, comme le dit Barthes : « je ne puis parler qu'en ramassant ce qui traîne dans la langue » (1978: 15) - ils inscrivent répétitivement, au cœur de l'énoncia-

148 Evoqué de façon plus détaillée dans Authier-Revuz (1995/2012 : 451-455), cet aspect bien connu du « rapport inquiet au langage » de Barthes, que celui-ci développe dans (Barthes, 1975), (Barthes, 1978), court dans toute son œuvre, (Barthes, 1981) et (Barthes, 1984) notamment.

149 Dont Barthes, s'inscrivant volontiers dans le sillage de Nietzsche, se plaît à noter ( $c f$. 1984 : 281, par exemple) l'usage concerté que celui-ci en faisait dans sa lutte contre la « maladie du langage » (cf. Haar, 1978) qu'est sa " grégarité ». 
tion des mots, par le questionnement, la suspicion dont ils l'affectent, ce « déboitement » d'un « langage qui se pense » apte, selon Barthes, à contrer, dans le mot énoncé, le sens « solidifié » de la doxa et à permettre que s'y « réveille » la « fraîcheur » d'un « frisson du sens ».

En chaque MAE se rejoue ponctuellement le fait que, dès lors que, depuis la position méta-énonciative qui est la sienne, je parle du déjà-dit qui est dans mon dire, je me soustrais d'autant à son emprise totale, il n'est plus en mesure de, strictement, me parler.

b) Du côté des pratiques de conquête d'une parole propre faisant appel à la RDA, c'est un genre, le pastiche ${ }^{150}$, fonctionnant « à la MAE », qui en donne l'exemple. Ce que J.-B. Pontalis, dans ses souvenirs tire de son expérience personnelle dans la vie courante :

Quelle ascèse plus efficace, pour qui voudrait être soi-même, que de savoir imiter ! Ainsi garde-t-on une chance, par le recours à des imitations conscientes et l'accentuation délibérée des traits du modèle, de ne pas être seulement l'écho de voix admirées. Les quelques personnes que je sais imiter [...] je suis à peu près sûr de n'en être pas la réplique involontaire (1986 : 157),

éclaire les enjeux de l'imitation dans l'écriture et, singulièrement, de l'usage, suivi et commenté, qu'en a fait Proust. La pratique mimétique d'écriture à la manière de, à la façon de permet à la fois de se démarquer de l'écriture autre et d'en afficher la maîtrise - la tonalité de la distanciation pouvant varier de la complicité amusée à la charge cruelle touchant la parodie.

Ce qui retient, dans le cas singulier des Pastiches ${ }^{151}$ de Proust, c'est qu'on peut, par un faisceau de traits, leur reconnaître un statut de " gammes » d'écriture, d'exercices de virtuosité stylistique pratiqués par un jeune écrivain en quête de sa voix propre :

- de façon exceptionnelle, ce n'est pas le pastiche d'un auteur, que compose Proust, mais une série de pastiches, comme une "suite de variations - Saint-Simonienne, Balzacienne, Flaubertienne... ${ }^{152}$ " - autour d'un même fait-divers contemporain, l'affaire Lemoine ;

- admiration et satire s'y mêlent en tonalités variables, mais, fait que Genette souligne comme « très caractéristique », " aucun de ces auteurs [n’a] suscité

150 Je renvoie au panorama qu’y consacre Genette (1982) dans son parcours de « la littérature au second degré ».

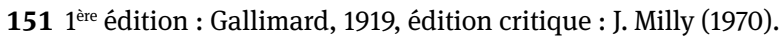

152 Genette (1982 : 131) ; variations passant aussi par Chateaubriand, Michelet, Renan, Régnier, Goncourt. 
chez Proust une condamnation ou une critique de sa singularité stylistique ${ }^{153}$;

- parus en volume en 1919, le travail d'écriture de ces pastiches appartient à une période de formation ${ }^{154}$, et c'est Proust lui-même qui évoque alors ${ }^{155}$ « la vertu purgative, exorcisante du pastiche », situant celle-ci dans sa capacité à faire se « dégager » la voix propre du pasticheur :

notre voix intérieure qui a été disciplinée [...] à suivre le rythme d'un Balzac, d'un Flaubert voudrait continuer à parler comme eux. Il faut la laisser faire un moment [...] faire un pastiche volontaire pour pouvoir après cela redevenir original, ne pas faire toute sa vie de pastiche involontaire ${ }^{156}$.

20 ans plus tard, lors de la publication des pastiches, Proust revient, dans une lettre $^{157}$, sur la visée - d'apparence plaisamment modeste - qu'il assigne au pastiche des maîtres :

[...] faire ouvertement [du Michelet ou du Goncourt] sous forme de pastiches pour redescendre à n'être plus que Marcel Proust quand j'écris des romans.

Comment mieux dire que, contrairement aux influences inaperçues qui « disciplinent », infléchissent, formatent notre « voix intérieure » en « parlant en elle », antinomique de cette autre pratique consciente de rapport à un discours autre qu'est le plagiat - dans lequel, le mensonge y côtoyant le trouble identitaire ${ }^{158}$, le sujet annule, « perd » sa voix propre -, le pastiche est, à visage découvert, une pratique dynamique et joueuse où l'on trouve sa voix. Et, contrairement au plagiaire, mélancolique, le pasticheur, Proust en fait la remarque ${ }^{159}$, est gai.

153 Ibid.p. 109.

154 Antérieure à 1908, date à laquelle ils paraissent, pour la plupart, dans le supplément littéraire du Figaro, en février-mars.

155 Dans le Contre Sainte-Beuve, publié de façon posthume en 1954, écrit entre 1895 et 1900, cité dans l'édition de la Pléiade, Gallimard, Paris, (1971).

156 Proust (1971) «À propos du “style” de Flaubert », Essais et articles, Bibl. de la Pléiade : $594-$ 595.

157 Lettre de 1919, à D. Fernandez, cité in Milly (1970 : 37).

158 Cf. Schneider (1985 : 270 sq., 292 sq., 300 sq.) sur le plagiat comme « absence de for intérieur », « maladie qui concerne les rapports du dehors et du dedans », où « à la place des transitions entre l'un et l'autre se produit un mode très archaïque de rapport à l'autre : l'ingestion » (idt, p. 304), réflexions illustrées notamment par les plagiats de Nerval à la fin de sa vie (p. 293 sq.). 159 Noté par Schneider (1985 : 340), qui renvoie à une lettre à D. Fernandez, citée in Le Divan, oct.-déc. 1948). 
Avec le pastiche s'achève cette collection disparate d' « histoires » de RDA et de parole propre : dans leur diversité - surprises dans la survenue dans le Dire de l'un d'un mot éprouvé comme d'un autre ; « remontées » en surface, du fond de l'enfance, depuis l'oubli, l'absence ou le figement où elles étaient retenues, de paroles qui peuvent, alors, être replacées comme « autres », dans le mouvement du Dire ; défense, pied à pied - mot à mot... - du Dire propre contre l'invasif déjà-dit ; dégagement d'une voix propre de l'emprise de celles, puissantes, qui l'entourent... - elles forment une variation sur le thème unique d'un parler d'un discours autre pour ne pas être parlé par lui, ou d'un passer par la RDA pour assurer sa parole propre ${ }^{160}$.

Du langage - de la greffe initiale dont il est l'objet à son fonctionnement en « extériorité interne »-, la parole émerge comme une question : celle, donc, source de plaintes, troubles, aventures, efforts... de faire " du dedans » avec « du dehors », d'énoncer une parole propre à partir de ce qui est Autre.

À travers les expériences langagières évoquées ci-dessus :

- celles où (2.3.2) dans la violence que le langage fait subir à ceux qu'il traverse, " parlant » à leur place, d'où solidairement se trouvent absents, autant la représentation d'un discours comme autre, que le sentiment d'une parole énoncée comme propre, la différence entre le propre et le non-propre n'a tout simplement pas cours ;

- celles où (2.3.4) la RDA, accompagnant la ré-émergence dans la parole d'un dire identifié comme autre, la dégage de l'emprise que celui-ci, insu, avait sur elle ;

- celles où (2.3.5) la RDA est, comme outil de défense ou de conquête d'une écriture propre, mise délibérément en œuvre face à la menace que fait peser sur elle la puissance de la grégarité ou la séduction des « grands modèles »;

la RDA et sa fonction dissimilatrice foncière de distinction d'une parole autre d'avec la parole en train de se faire, apparaît comme ayant partie liée avec la possibilité pour le sujet d'énoncer une parole comme propre. Dans les trois cas, ce sont les frontières, limites, bords, auxquels la parole peut (ou non) s'assurer comme

$160 \mathrm{Si}$, dans les cas particuliers évoqués ici, la MAE - où le rapport dissimilateur à l'autre d'inscrit au plus intime du dire de l'un, par la mention passant au travers des mots dont il fait usage - est la forme de RDA majoritairement convoquée, c'est (cf. chapitre suivant) la RDA dans son ensemble qui s'articule avec la « question » de la parole propre. 
propre qui sont en jeu : bords manquants d'une non-parole sans autre, bords retrouvés, libérateurs, avec un autre, bords conquis d'une écriture propre dans son environnement.

L'observation, dans ces dires variés des rencontres entre RDA et parole énoncée comme propre (fût-ce sur le mode de leur commune absence) met sur le chemin de la fonction énonciative « ordinaire» de la RDA dans le dire : celle, sur le fond, réel, d'un dehors au dedans du dire, d'assurer la parole dans des frontières, limites, bords qui, pour être imaginaires, lui sont vitalement nécessaires. 